\title{
Spin-polarized hydrogen and its isotopes: a rich class of quantum phases
}

\section{(Review Article)}

\author{
I. Bešlic ${ }^{1,2}$, L. Vranješ Markić ${ }^{2}$, and J. Boronat ${ }^{1}$ \\ ${ }^{1}$ Departament de Física i Enginyeria Nuclear, Campus Nord B4-B5 \\ Universitat Politècnica de Catalunya, E-08034 Barcelona, Spain \\ E-mail: jordi.boronat@upc.edu \\ ${ }^{2}$ Faculty of Science, University of Split, HR-21000 Split, Croatia
}

Received March 4, 2013

\begin{abstract}
We review the recent activity in the theoretical description of spin-polarized atomic hydrogen and its isotopes at very low temperatures. Spin-polarized hydrogen is the only system in nature that remains stable in the gas phase even in the zero temperature limit due to its small mass and weak interatomic interaction. Hydrogen and its heavier isotope tritium are bosons, the heavier mass of tritium producing a self-bound (liquid) system at zero temperature. The other isotope, deuterium, is a fermion with nuclear spin one making possible the study of three different quantum systems depending on the population of the three degenerate spin states. From the theoretical point of view, spin-polarized hydrogen is specially appealing because its interatomic potential is very accurately known making possible its precise quantum many-body study. The experimental study of atomic hydrogen has been very difficult due to its high recombination rate, but it finally led to its Bose-Einstein condensate state in 1998. Degeneracy has also been observed in thin films of hydrogen adsorbed on the ${ }^{4}$ He surface allowing for the possibility of observing the Berezinskii-Kosterlitz-Thouless superfluid transition.
\end{abstract}

PACS: 64.60. Bd General theory of phase transitions;

67.63.Gh Atomic hydrogen and isotopes;

67.85.Jk Other Bose-Einstein condensation phenomena.

Keywords: spin-polarized hydrogen, Berezinskii-Kosterlitz-Thouless superfluid transition.

\section{Contents}

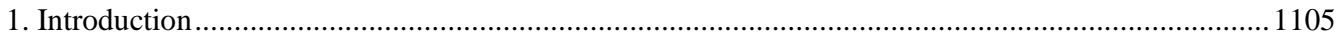

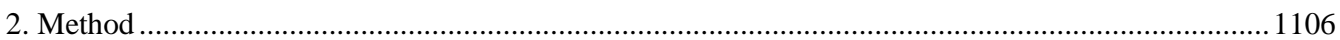

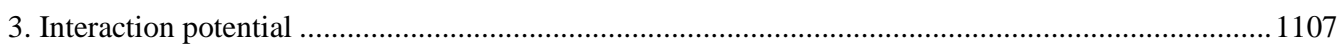

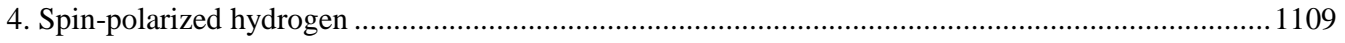

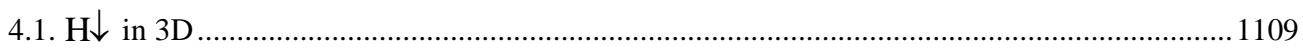

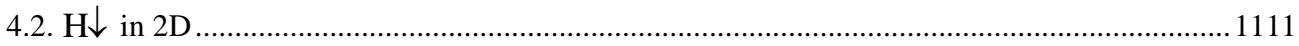

4.3. Spin-polarized hydrogen adsorbed on the surface of liquid helium .............................................1112

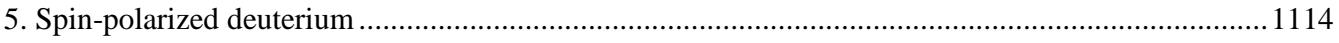

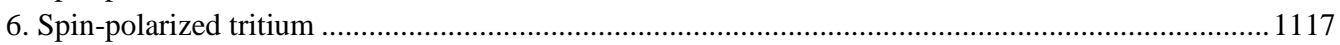

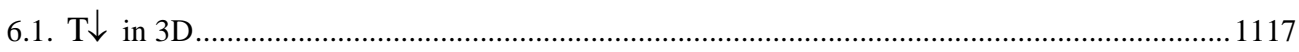

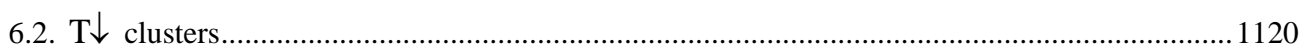

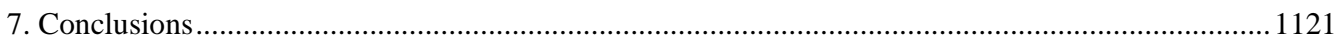

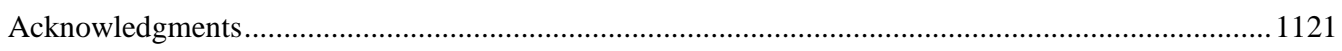

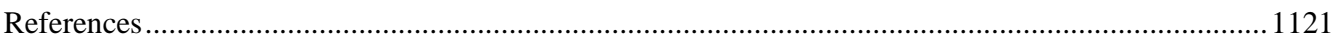




\section{Introduction}

In the limit of temperatures close to the absolute zero some systems show macroscopic quantum phenomena such as superfluidity and superconductivity. The physics of liquid helium $\left({ }^{4} \mathrm{He}\right.$ and ${ }^{3} \mathrm{He}$ ) has been investigated for a long time, and it was shown that relevant properties of the system are direct manifestations of its extreme quantum nature and, in particular, quantum statistics. The surprising findings of the novel phenomena, such as superfluidity and superconductivity, as well as supersolidity, enforced further theoretical and experimental effort in the field of strongly correlated quantum many-body systems.

Even though electron-spin-polarized hydrogen $(\mathrm{H} \downarrow)$ can not be found in nature, it can be produced experimentally. For a very long time, this system puzzled the theoretical community and much theoretical effort was invested in calculations of the accurate interaction potential for two $\mathrm{H} \downarrow$ atoms in the triplet $b^{3} \Sigma_{u}^{+}$state. Finally, in 1965 Kolos and Wolniewicz [1] provided results for a very precise $\mathrm{H} \downarrow-\mathrm{H} \downarrow$ interaction potential. Their result revealed that the interaction between $\mathrm{H} \downarrow$ atoms is even weaker than the helium-helium one. It was clear already from the first theoretical investigations that electron-spin-polarized hydrogen could be the only system in nature possessing the unique property of remaining in the gaseous state at zero temperature [2,3]. Having in mind the very light mass of $\mathrm{H}$ atoms and its very shallow interaction potential, and using the quantum parameter $\eta=\hbar^{2} /\left(m \varepsilon \sigma^{2}\right)$ as a measure of the quantum character of a system, Stwalley and Nosanow [4] concluded that spin-polarized atomic hydrogen could exhibit even more extreme quantum behavior than helium does. In fact, they proposed gas $\mathrm{H} \downarrow$ as an ideal candidate for achieving a Bose-Einstein condensate (BEC). That prediction led the future interest of both, the theoretical and experimental communities.

From the experimental point of view, the extensive $\mathrm{H} \downarrow$ study started in Amsterdam in 1980 when Silvera and Walraven managed to stabilize a very dilute gas of spinpolarized hydrogen for the first time [5]. A long experimental journey preceded the final realization of a BEC state in $\mathrm{H} \downarrow$. At last in 1998 Fried et al. [6] used successfully a wall-free confinement and evaporative cooling, enforced with a radio-frequency ejection technique, to achieve the necessary conditions for BEC. In their experiment, a gas of atomic hydrogen was confined in a cylindrically symmetric magnetic trap, and cooled to $120 \mu \mathrm{K}$ by evaporation, i.e., by allowing some of the atoms escape by lowering the confining potential at one end of the trap [6,7]. For temperatures below about $120 \mu \mathrm{K}$ this kind of cooling appeared to be not satisfactory enough since the high-energy atoms near the centre of the trap could loose their energy by collisions before escaping from the trap. Thus, the evaporation was not effective for atoms with high energy because the escape time of those atoms was very long when the temperature was reduced. The gain of evaporation efficiency was achieved using a radio-frequency magnetic field that flipped the spins of atoms in the trap, wherever the resonance condition was fulfilled for the trapping magnetic field. The atoms with reversed spins were not confined any more, and they were allowed to leave the trap and to enforce in that way the prerequisite conditions for a BEC realization. A radio-frequency ejection technique enabled cooling enhancement and when the atoms were cooled below $50 \mu \mathrm{K}$ the indication of a BEC in $\mathrm{H} \downarrow$ appeared.

Another very important experimental achievement was also accomplished in 1998 by Safonov et al. [8] when they realized a quasi-condensate of nearly two-dimensional $\mathrm{H} \downarrow$ adsorbed on the surface of superfluid ${ }^{4} \mathrm{He}$. Their result provided the best example of formation of a stable nearly two-dimensional quantum Bose gas. Further experimental investigations in this area have been addressed to the observation of the Berezinskii-Kosterlitz-Thouless (BKT) superfluid transition. The superfluid transition in a twodimensional quantum Bose gas is predicted to appear when the quantum degeneracy parameter $\sigma \Lambda^{2} \simeq 4$ [9], where $\sigma$ is the surface density and $\Lambda$ is the thermal de Broglie wave-length. In order to reach quantum degeneracy in twodimensional $\mathrm{H} \downarrow$ gas two methods of local compression have been used: magnetic and thermal compressions [10]. The first one enables the highest value of the quantum degeneracy parameter ( $\sigma \Lambda^{2} \simeq 9$ ), but has the disadvantage of not being able to ensure the direct study of the compressed surface gas because of the large field gradients. The second one allows direct observation of the surface gas, but provides a relatively small value of the quantum degeneracy parameter ( $\sigma \Lambda^{2} \simeq 1.5$ ), leaving thus the scientific community still without experimental evidence of the BKT superfluid transition in hydrogen.

Recently, spin-polarized deuterium has been trapped for the first time using 24-stage Zeeman decelerator [11] at a temperature of $100 \mathrm{mK}$, representing a first step towards reaching quantum degeneracy. The same technique has been used to trap hydrogen as well [12] and could also be used to trap tritium.

From the theoretical point of view, the development of the theoretical methods in the last decades was genuinely motivated by the successful experimental measurements. In addition to the early theoretical work dedicated to $\mathrm{H} \downarrow$ [2,3,13-15], that was done using different interatomic potentials and different variational methods, there was a respectable amount of theoretical work dedicated to investigation of its isotopes, spin-polarized deuterium (D $\downarrow$ )

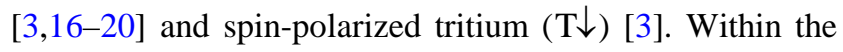
Born-Oppenheimer approximation, electron-spin-polarized hydrogen atoms behave as effective bosons, as well as $\mathrm{T} \downarrow$, whereas $\mathrm{D} \downarrow$ has Fermi symmetry [21]. A rich class of quantum phases exist in $\mathrm{D} \downarrow$, depending on how $\mathrm{D} \downarrow$ atoms are distributed with respect to the available nuclear spin 
states. However, the most theoretically studied $\mathrm{D} \downarrow$ systems are those that are defined with equal occupation of the nuclear spin states ( $\left.\mathrm{D} \downarrow_{1}, \mathrm{D} \downarrow_{2}, \mathrm{D} \downarrow_{3}\right)$.

Quantum Monte Carlo (QMC) methods are standard investigation tools for studying correlated quantum systems, probably the most accurate ones from the quantitative point of view. Recent theoretical activity in the study of spin-polarized atomic hydrogen and its isotopes has appeared after Jamieson et al. [22] recalculated and extended to larger interparticle distances the triplet pair potential $b^{3} \Sigma_{u}^{+}$. The ground-state properties of $\mathrm{H} \downarrow, \mathrm{D} \downarrow$ and $\mathrm{T} \downarrow$ have been investigated using the newest triplet pair potential $b^{3} \Sigma_{u}^{+}$and the diffusion Monte Carlo (DMC) method [23-25]. Since previously obtained results used variational methods, the recent use of the DMC method in the study of spin-polarized atomic hydrogen and its isotopes brought up theoretical descriptions of those systems to an improved level. The gas phase of $\mathrm{H} \downarrow$ was investigated from the very dilute regime up to the density above which the system crystallizes $[23,26]$. In order to determine the density and the pressure at which the system experiences a gas-solid transition at $T=0$, the solid phase of $\mathrm{H} \downarrow$ was also investigated up to higher pressure. A theoretical investigation of $\mathrm{H} \downarrow$ using QMC methods has been completed with a study of a thin layer of adsorbed on top of the surface of superfluid ${ }^{4} \mathrm{He}$ [27]. The investigations were further extended to the study of spin-polarized tritium. Its liquid and solid phase were studied, and the conditions under which a liquid-solid phase transition occurs in the limit of zero temperature were determined [24]. The large $\mathrm{T} \downarrow$ clusters were also studied and from their ground-state properties several liquid $\mathrm{T} \downarrow$ ground-state properties were extrapolated [28]. As the only representative with the Fermi statistics, spinpolarized deuterium was recently investigated. The groundstate properties of $\mathrm{D} \downarrow$ at zero temperature were obtained within the fixed-node approximation, and the influence of the backflow correlations on the ground-state energy of the systems was explored. The liquid phases of $\mathrm{D} \downarrow_{3}$ and $\mathrm{D} \downarrow_{2}$ were characterized, while the liquid-gas coexistence region of $\mathrm{D} \downarrow_{1}$ was also determined [25,29].

The rest of the paper is organized as follows: in Sec. 2 the QMC methods and the trial wave functions used for importance sampling are briefly described, and in Sec. 3 the interatomic potential is reviewed. The results are presented in the Secs. 4, 5 and 6. Sec. 4 is dedicated to $\mathrm{H} \downarrow$, where subsections cover the results concerning the 3D study, the 2D study and the study of $\mathrm{H} \downarrow$ adsorption on the surface of liquid ${ }^{4} \mathrm{He}$. In Sec. 5 the ground-state properties of three $\mathrm{D} \downarrow$ species are presented, and $\mathrm{T} \downarrow$ ground-state properties are reported in Sec. 6. The main conclusions of this review are summarized in the last section.

\section{Method}

In this section the Monte Carlo methods which are commonly used for zero temperature calculations are brief- ly discussed. Most theoretical studies using Monte Carlo methods begin with the application of the variational Monte Carlo (VMC) methodology. As it is well know from the variational principle of Quantum Mechanics, the energy calculated using an approximate wave function $\Psi_{T}$ (not orthogonal to the ground-state wave function) is always an upper bound to the exact ground-state energy $E_{0}$, i.e., $E_{V M C} \geq E_{0}$. A homogeneous system of atoms interacting via a central interatomic potential $V\left(r_{i j}\right)$ is described with Hamiltonian

$$
H=-\frac{\hbar^{2}}{2 m} \sum_{i=1}^{N} \nabla_{i}^{2}+\sum_{i<j}^{N} V\left(r_{i j}\right)
$$

The Hamiltonian of a thin layer of adsorbed $H \downarrow$ on top of the surface of superfluid ${ }^{4} \mathrm{He}$ is given by

$$
\begin{aligned}
H=-\frac{\hbar^{2}}{2 m_{\mathrm{He}}} & \sum_{I=1}^{N_{\mathrm{He}}} \nabla_{I}^{2}-\frac{\hbar^{2}}{2 m_{\mathrm{H}}} \sum_{i=1}^{N_{\mathrm{H}}} \nabla_{i}^{2}+\sum_{1=I<J}^{N_{\mathrm{He}}} V_{\mathrm{He}-\mathrm{He}}\left(r_{I J}\right)+ \\
& +\sum_{1=i<j}^{N_{\mathrm{H}}} V_{\mathrm{H}-\mathrm{H}}\left(r_{i j}\right)+\sum_{1=I, i}^{N_{\mathrm{He}}, N_{\mathrm{H}}} V_{\mathrm{He}-\mathrm{H}}\left(r_{I i}\right)
\end{aligned}
$$

where normal and capital indices stand for ${ }^{4} \mathrm{He}$ and $\mathrm{H} \downarrow$ atoms, respectively.

In accordance with the VMC methodology, system configurations $\mathbf{R} \equiv\left(\mathbf{r}_{1}, \ldots, \mathbf{r}_{N}\right)$, which are called walkers, are being generated according to the probability density function $\left|\Psi_{T}(\mathbf{R})\right|^{2}$ and the energy $E_{V M C}$ is obtained as the mean value of the local energy $E_{L}(\mathbf{R}) \equiv H \Psi_{T}(\mathbf{R}) / \Psi_{T}(\mathbf{R})$. The optimization of the $\Psi_{T}$ variational parameters improves the quality of the upper-bound of the ground-state energy of the system. The system configurations obtained with the VMC method, as well as the optimal $\Psi_{T}$ variational parameters, are used as input for the next phase of the investigations, i.e., in the diffusion Monte Carlo calculations.

The trial wave function used in simulations of gas $\mathrm{H} \downarrow$, liquid $\mathrm{T} \downarrow$ and $\mathrm{T} \downarrow$ clusters is of the Jastrow form

$$
\psi_{T}(\mathbf{R})=\psi_{J}(\mathbf{R})=\prod_{i<j}^{N} f(r),
$$

where the two-body correlation functions that describe the dynamical correlations are given in Table 1 . The solid phases are studied using the Nosanow-Jastrow model [30]

$$
\psi_{T}(\mathbf{R})=\psi_{N J}(\mathbf{R})=\psi_{J}(\mathbf{R}) \prod_{i}^{N} h\left(r_{i I}\right),
$$

where a localization function that links every particle $i$ to a fixed lattice point $I$ is taken in a Gaussian form $h(r)=\exp \left(-\alpha r^{2} / 2\right)$. 
Table 1. Two-body correlation functions $f(r)$ used to describes the dynamical correlations between particles

\begin{tabular}{c|c}
\hline \hline Particle & $f(r)$ \\
\hline \hline $\mathrm{H} \downarrow, \mathrm{T} \downarrow$ & $\exp \left[-b_{1} \exp \left(-b_{2} r\right)\right]$ \\
$\mathrm{D} \downarrow$ & $\exp \left[-\frac{1}{2}\left(\frac{b}{r}\right)^{5}\right]$ \\
$(\mathrm{T} \downarrow)_{N}$ & $\exp \left[-\left(\frac{b}{r}\right)^{5}-s r\right], 20 \leq N \leq 60$ \\
& $\begin{array}{c}\exp \left[-\left(\frac{b}{r}\right)^{5}-s r^{2}\right], 80 \leq N \leq 320 \\
\mathrm{H} \downarrow \text { on }{ }^{4} \mathrm{He}\end{array}$ \\
& $\exp \left[-b_{1} \exp \left(-b_{2} r\right)\right], \mathrm{H} \downarrow-\mathrm{H} \downarrow$ \\
& $\exp \left[-\frac{1}{2}\left(\frac{b}{r}\right)^{5}\right],{ }^{4}{ }^{\mathrm{He}}-{ }^{4} \mathrm{He},{ }^{4} \mathrm{He}-\mathrm{H} \downarrow$ \\
\hline \hline
\end{tabular}

In the case of bulk $\mathrm{D} \downarrow$, the antisymmetric wave function $\psi_{A}(\mathbf{R})$ which introduces the Fermi statistics has to be introduced in $\psi_{T}(\mathbf{R})$, and thus the trial wave function has the form

$$
\psi_{T}(\mathbf{R})=\psi_{A}(\mathbf{R}) \psi_{J}(\mathbf{R})=\psi_{A}(\mathbf{R}) \prod_{i<j}^{N} f(r) .
$$

The two-body correlation function $f(r)$ used in bulk $\mathrm{D} \downarrow$ is reported in Table 1, and $\psi_{A}(\mathbf{R})$ is modelled with a Slater determinant for $D \downarrow_{1}$, and the product of two and three Slater determinants in the case of $\mathrm{D} \downarrow_{2}$ and $\mathrm{D} \downarrow_{3}$, respectively. In all cases the Slater determinants were constructed using a single-particle plane-wave orbitals that correspond to the exact wave function of the Fermi sea, $\varphi_{\alpha_{i}}\left(\mathbf{r}_{j}\right)=\exp \left(i \mathbf{k}_{\alpha_{i}} \mathbf{r}_{j}\right)$. In addition, the trial wave function was improved by introducing momentum-dependent correlations in the antisymmetric wave function. The backflow correlations were modelled in a similar way as in the work by Panoff and Clark [19], but omitting the long-range term $\left(\lambda_{B}^{\prime} / r^{3}\right)$, i.e., in the following way:

$$
\tilde{\mathbf{r}}_{j}=\mathbf{r}_{j}+\lambda_{B} \sum_{k \neq j} \exp \left[-\left(\frac{r_{j k}-r_{B}}{\omega_{B}}\right)^{2}\right]\left(\mathbf{r}_{j}-\mathbf{r}_{k}\right),
$$

where $\lambda_{B}, r_{B}$ and $\omega_{B}$ are variational parameters.

The study of a thin layer of adsorbed $\mathrm{H} \downarrow$ on top of the surface of superfluid ${ }^{4} \mathrm{He}$ is carried out using the trial wave function

$$
\begin{gathered}
\psi_{T}(\mathbf{R})=\psi_{J}(\mathbf{R}) \Phi(\mathbf{R})= \\
=\prod_{1=I<J}^{N_{\mathrm{He}}} f_{\mathrm{He}}\left(r_{I J}\right) \prod_{1=i<j}^{N_{\mathrm{H}}} f_{\mathrm{H}}\left(r_{i j}\right) \prod_{1=I, i}^{N_{\mathrm{He}}, N_{\mathrm{H}}} f_{\mathrm{He}-\mathrm{H}}\left(r_{I i}\right) \Phi(\mathbf{R}) .
\end{gathered}
$$

The given ansatz (7) includes products of $f(r)$ for different pairs of particles (Table 1), and the function $\Phi(\mathbf{R})$ is defined with the products of the one-body correlations that describe the slab geometry confinement in the system

$$
\Phi(\mathbf{R})=\prod_{I=1}^{N_{\mathrm{He}}} h_{\mathrm{He}}\left(z_{I}\right) \prod_{i=1}^{N_{\mathrm{H}}} h_{\mathrm{H}}\left(z_{i}\right) .
$$

The diffusion Monte Carlo method is used to solve stochastically the Schrödinger equation written in imaginary time,

$$
-\hbar \frac{\partial \Psi(\mathbf{R}, t)}{\partial t}=\left(H-E_{r}\right) \Psi(\mathbf{R}, t)
$$

in which constant $E_{r}$ acts as a reference energy. In order to introduce the importance sampling, the Schrödinger equation has to be rewritten in terms of the mixed distribution $\Psi(\mathbf{R}, t) \psi_{T}(\mathbf{R})$. The diffusion process is governed with the auxiliary wave function $\psi_{T}(\mathbf{R})$ which acts as a guiding wave function towards the ground state. In the limit $t \rightarrow \infty$ only the lowest energy eigenfunction survives (not orthogonal to $\psi(\mathbf{R})$ ), and then the sampling of the ground state for a $\mathrm{N}$-body bosonic system is effectively achieved. In addition, for Fermi systems one has to deal with the sign problem, and that is usually circumvented by working within the fixed-node approximation. The fixednode approximation is based on the idea of imposing common $\Psi(\mathbf{R}, t)$ and $\psi(\mathbf{R})$ nodes, i.e., it is assumed that those functions change the sign together. Since the trial wave function $\psi(\mathbf{R})$ determines the nodes, the obtained results depend on the accuracy of the $\psi(\mathbf{R})$ nodes, with respect to the real ones. In the way, the obtained fixednode energies are upper bounds to the exact ground-state energy.

In order to avoid the dependence of the results on the possible systematic errors, a careful analysis was carried out. Statistical uncertainty was reduced by using the required number of walkers for which the DMC results did not depend on the particular number employed in the simulations. The DMC method, accurate to the second order in the time step $\Delta t$, was implemented in the codes [31], to minimize the dependence on the finite time steps that were used during the time evolution of the system. The forward walking strategy was used to ensure the pure estimation of the observables [32], and to reduce any bias coming from the choice of the guiding function $\psi_{T}(\mathbf{R})$. Finally, simulations of bulk systems with a finite number of particles require of a size-dependence analysis. To this end, tail corrections were properly included, as well as Fermi corrections in case of Fermi systems [25,29,33,34].

\section{Interaction potential}

Spin-polarized hydrogen atoms interact via the triplet potential $b^{3} \Sigma_{u}^{+}$, calculated with high precision by Kolos and Wolniewicz (KW) in 1965 [1]. The simplicity of the $\mathrm{H}$ atom makes possible to calculate this potential in an essentially exact way. More recently, it has been recalculated up to larger interatomic distances by Jamieson, Dalgarno and Wolniewicz (JDW) [22]. The differences between the KW 


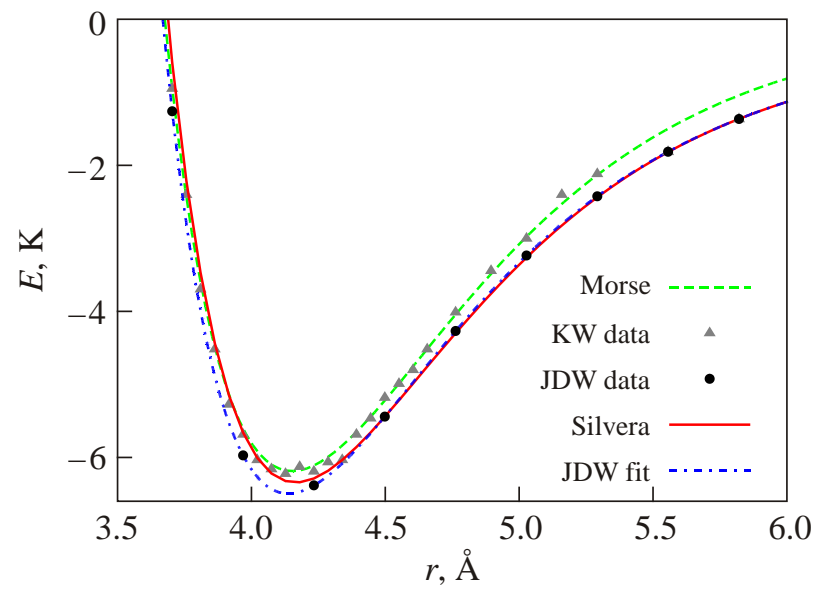

Fig. 1. (Color online) $\mathrm{H} \downarrow-\mathrm{H} \downarrow$ interatomic potentials.

and JDW potentials, in the range where they can be compared, are rather small, as shown in Fig. 1. The addition of mass-dependent adiabatic corrections, which have been calculated by Kolos and Rychlewski [35], to the JDW potential can not be discerned in Fig. 1, and we can say that the interaction potential between all possible hydrogen isotopes is essentially the same.

From 60ties to the beginning of this century, only the KW potential was used in the study of gas $\mathrm{H} \downarrow$. Usually, an analytic form was assumed and then the free parameters of the model were fitted to reproduce the KW data. In this way, Etters et al. [3] used a Morse potential whereas Silvera and Goldman [36] proposed a form which is similar to the ones used for He-He potentials. The results of these models are also plotted in Fig. 1. In the last years, in most studies of spin-polarized hydrogen and its isotopes the JDW interaction has been used [23-29,37-41], which is plotted as a solid line in Fig. 1. The JDW data are smoothly connected with the long-range behavior of the $\mathrm{H} \downarrow-\mathrm{H} \downarrow$ potential as calculated by Yan et al. [42]. The JDW potential is slightly deeper than $\mathrm{KW}$ with a minimum $\varepsilon=-6.49 \mathrm{~K}$ at a distance $r_{m}=4.14 \AA$, and a core diameter of $3.67 \AA$.

The influence of the potential on the energy of the gas at small densities was studied in Ref. 23. The comparison was done using VMC calculations and the KW and JDW potentials. From Fig. 2, it is clear that the JDW energies are below the KW ones in all the density range, reflecting the slightly deeper well of the JDW potential. In the calculation with the Morse potential adjusted by Etters et al. [3] to the KW data the VMC energies are significantly worse than the KW ones. This manifests the difficulties in fitting a functional form to the $a b$ initio KW data; the Morse potential is a bit more repulsive than $\mathrm{KW}$ and therefore the energies are higher. As a matter of comparison, results from previous calculations are shown in Fig. 2. The results from Etters et al. [3] using the Morse potential are in nice agreement with the VMC results using the same potential.

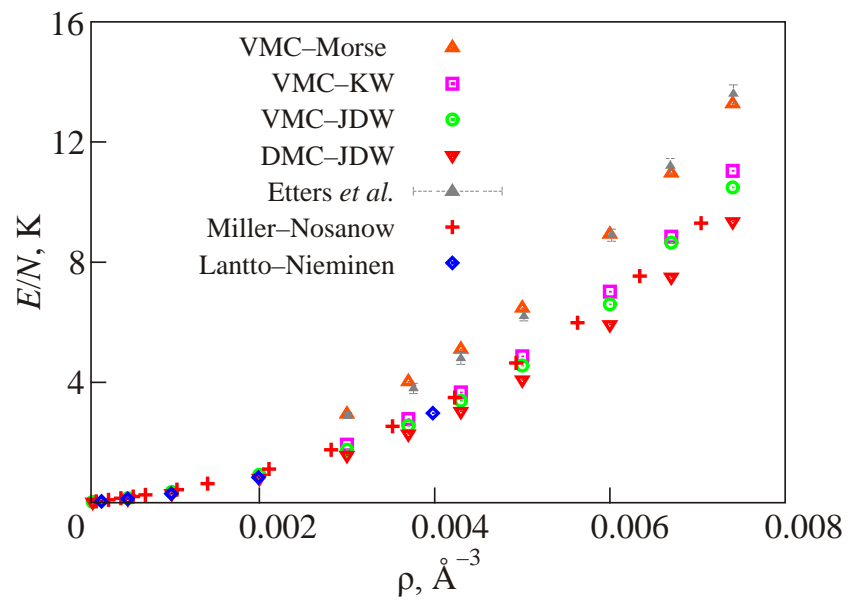

Fig. 2. (Color online) Influence of the interatomic potential on the equation of state of gas $\mathrm{H} \downarrow$.

The variational results of Miller and Nosanow [13] used the $\mathrm{KW}$ data and are in close agreement with the VMC results using the same interaction. Finally, results of Lantto and Nieminen [14] are also reported; they used the KW potential and performed an Euler-Lagrange-HNC calculation. Their results, restricted to very low densities, are slightly better than the VMC results due to their use of an optimized Jastrow factor. All these variational results, are compared in the same figure with the DMC results using the JDW interaction. As expected, the DMC results are below the VMC ones in all the density range with a difference that increases with $\rho$, a predictable feature arising from the fact that the Jastrow factor in the trial wave function (3) corresponds to an analytical form which approximates the wave function solution of the two-body problem.

In the theoretical study of $\mathrm{H} \downarrow$ adsorbed on the surface of liquid helium two other interaction potentials are needed, ${ }^{4} \mathrm{He}-{ }^{4} \mathrm{He}$ and $\mathrm{H} \downarrow-{ }^{4} \mathrm{He}$. For the ${ }^{4} \mathrm{He}-{ }^{4} \mathrm{He}$ interactions one of the most used models, which was shown to accurately predict experimental bulk helium data is the Aziz HFD-B(He) interaction potential [43]. Several models are available for $\mathrm{H} \downarrow-{ }^{4} \mathrm{He}$ interactions; the recent work on

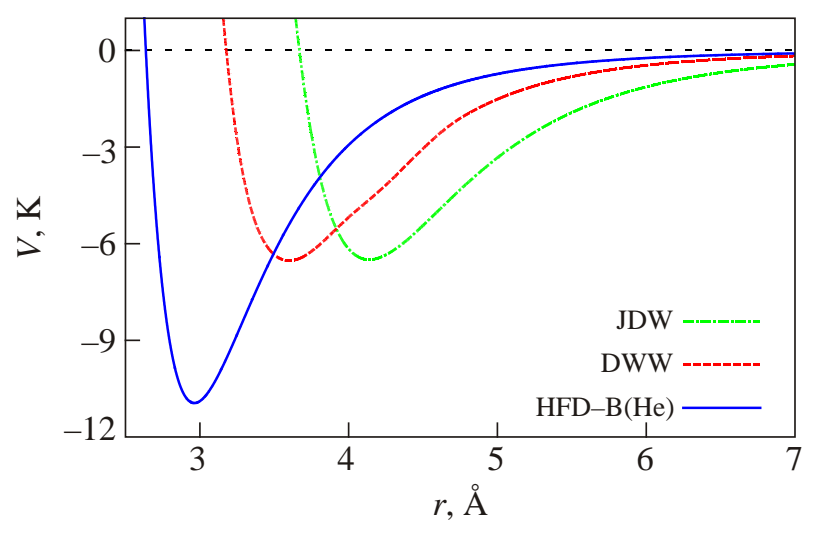

Fig. 3. (Color online) Comparison among interaction potentials between ${ }^{4} \mathrm{He}-{ }^{4} \mathrm{He}$ (HFD-B(He)) [43], ${ }^{4} \mathrm{He}-\mathrm{H} \downarrow$ (DWW) [44] and $\mathrm{H} \downarrow-\mathrm{H} \downarrow$ (JDW) [22] atoms. 
$\mathrm{H} \downarrow$ adsorbed on liquid helium [27] used the model by Das et al. [44], which we compare in Fig. 3 with HFD and JDW potentials. Of all the interaction potentials, the $\mathrm{H} \downarrow-\mathrm{H} \downarrow$ one has the largest core and the most attractive long-range part.

\section{Spin-polarized hydrogen}

\section{1. $\mathrm{H} \downarrow$ in $3 D$}

The gas phase of spin-polarized $\mathrm{H}$ at very low densities has been in the focus of research for many years due to the expected and later realized Bose-Einstein condensation at low temperatures. From the theoretical perspective, $\mathrm{H} \downarrow$ is even more appealing than alkali gases because the interatomic interaction between $\mathrm{H} \downarrow$ atoms is very well known, as we have commented in the previous subsection. The equation of state of a bosonic gas becomes universal at sufficiently low densities, when expressed in terms of the gas parameter $x=\rho a^{3}$, with $a$ the $s$-wave scattering length. It is given by

$$
\left(\frac{E}{N}\right)=4 \pi x\left(1+\frac{128}{15 \sqrt{\pi}} x^{1 / 2}\right),
$$

where the first term is the mean-field result [45], and the second is the Lee-Huang-Yang correction [46]; the energy per particle is written in units of $\hbar^{2} /\left(2 m a^{2}\right)$.

The recent DMC study of hydrogen at $T=0$ from Ref. 23 confirmed the agreement with the universal equation of state at low densities. The energy per particle of gas $\mathrm{H} \downarrow$ was compared to the universal equation of phase (10) and to the DMC results for a hard-sphere (HS) gas from Ref. 47. For that purpose, the $s$-wave scattering length of the JDW potential was determined to be $a=0.697 \AA$ in agreement with previous calculations $[37,48]$. The results, presented in Fig. 4, show that the equation of state of gas $\mathrm{H} \downarrow$ coincides with both the analytic law (10) and the HS results up to $x \simeq 10^{-4}$, in agreement with the range of un-

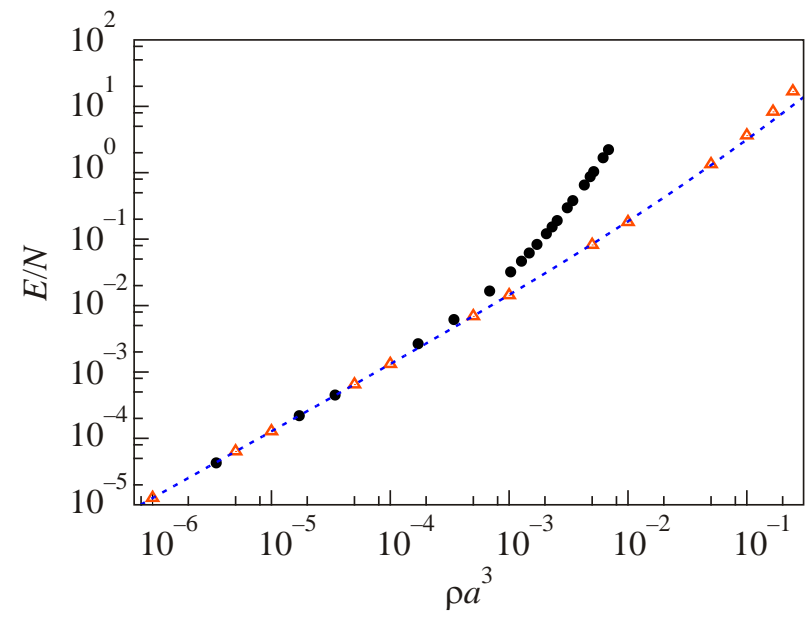

Fig. 4. (Color online) Equation of state of gas $\mathrm{H} \downarrow$ as a function of the gas parameter $\rho a^{3}$ in units of $\hbar^{2} /\left(2 m a^{2}\right.$ ) (solid points). The triangles correspond to a HS gas [47] and the line to Eq. (10). iversality determined in Ref. 47. Beyond this value, the energies of bulk $\mathrm{H} \downarrow$ clearly separate from Eq. (10), increasing with $x$ faster than for the HS gas.

Recently, hydrogen gas at low densities was also studied by Joudeh et al. using the Brueckner-Bethe-Goldstone theory [38]. Due to the approximate nature of the method, their results lie slightly below the universal equation of state.

At very low densities, a universal behavior in terms of the gas parameter $x$ is also known for the condensate fraction $n_{0}$, i.e., the fraction of particles occupying the zeromomentum state. In Fig. 5, results for the condensate fraction of gas $\mathrm{H} \downarrow$ (Ref. 23) are compared with the Bogoliubov formula [45]

$$
n_{0}=1-\frac{8}{3 \sqrt{\pi}} x^{1 / 2}
$$

and DMC results [47] for $n_{0}$ in the HS system. In the DMC simulations the condensate fraction is obtained from the long-range behavior of the one-body density matrix $\rho(r), n_{0}=\lim _{r \rightarrow \infty} \rho(r)$. As one can see, the three results are coincident up to $x \simeq 10^{-4}$, the same value observed for the energy in Fig. 4. Both HS and $\mathrm{H} \downarrow$ show a faster decrease with $x$ than the law (11), the departure from it being significantly larger for hydrogen, in agreement with the same feature observed in Fig. 4 for the energy. The condensate fraction was determined for the whole range of densities, up to freezing. The obtained set of data is well reproduced using the functional form

$$
n_{0}(\rho)=1-\frac{8}{3 \sqrt{\pi}}\left(\rho a^{3}\right)^{1 / 2}-b_{1} \rho a^{3}-b_{2}\left(\rho a^{3}\right)^{2}-b_{3}\left(\rho a^{3}\right)^{5 / 2} .
$$

The values of the parameters in Eq. (12) are $b_{1}=504(5)$, $b_{2}=-1.254(49) \cdot 10^{5}, b_{3}=8.54(55) \cdot 10^{5}$, and $a$ is the scattering length.

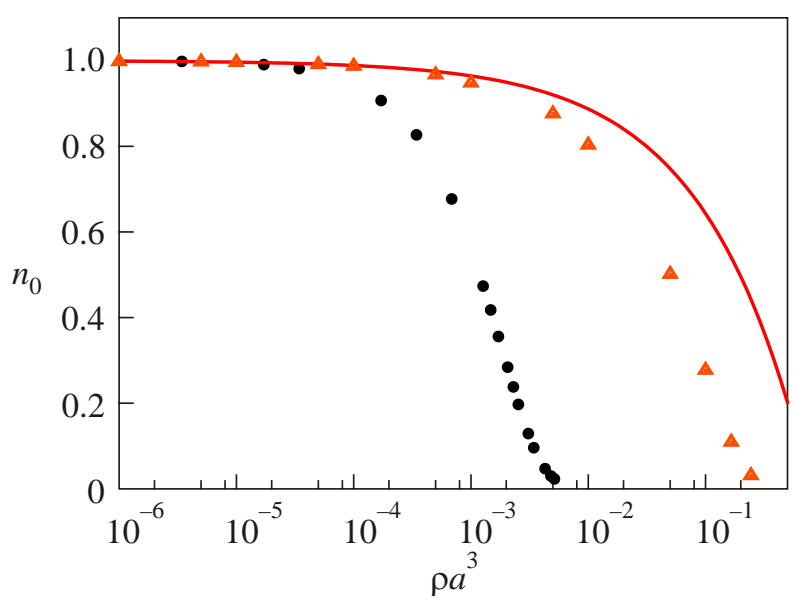

Fig. 5. (Color online) Condensate fraction of gas $\mathrm{H} \downarrow$ as a function of the gas parameter $\rho a^{3}$ (solid points). The triangles correspond to a HS gas [47] and the line to the Bogoliubov approximation (11). 

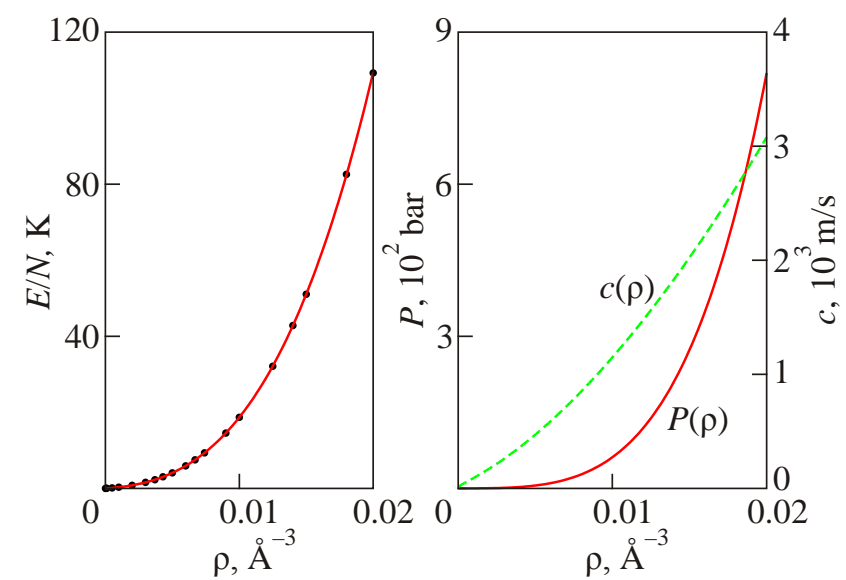

Fig. 6. (Color online) Left: Energy per particle of gas $\mathrm{H} \downarrow$ (solid circles) as a function of the density $\rho$. The solid line corresponds to the fit to the DMC energies using Eq. (13). The error bars of the DMC energies are smaller than the size of the symbols. Right: Pressure and speed of sound of gas $\mathrm{H} \downarrow$ as a function of the density. Left (right) scale corresponds to pressure (speed of sound).

The gas equation of state was also obtained for the whole range of densities up to freezing. Results are well parameterized by a polynomial form ( $e \equiv E / N)$

$$
e(\rho)=e_{1} \rho+e_{2} \rho^{2}+e_{3} \rho^{3}+e_{4} \rho^{4}
$$

shown as a solid line on top of the DMC results in Fig. 6. The best set of parameters is: $e_{1}=217.0(1.9) \mathrm{K} \cdot \AA$, $e_{2}=7.76(9) \cdot 10^{4} \quad \mathrm{~K} \cdot \AA^{2}, \quad e_{3}=8.23(12) \cdot 10^{6} \quad \mathrm{~K} \cdot \AA^{3}, \quad$ and $e_{4}=5.1(5) \cdot 10^{7} \mathrm{~K} \cdot \AA^{4}$, the figures in parenthesis being the statistical uncertainties.

Knowledge of the equation of state enables calculation of the pressure and the speed of sound using their thermodynamic definition

and

$$
P(\rho)=\rho^{2}\left(\frac{\partial e}{\partial \rho}\right),
$$

$$
c^{2}(\rho)=\frac{1}{m}\left(\frac{\partial P}{\partial \rho}\right) .
$$

Results obtained in Ref. 23 are presented in Fig. 6.

Relevant structural quantities were determined using DMC simulations as well [23], the two-body radial distribution function $g(r)$ and its Fourier transform, the static structure function $S(k)$. The evolution of $g(r)$ with density for the gas $\mathrm{H} \downarrow$ is shown in Fig. 7. At the smallest density reported, $g(r)$ is a monotonic function with the corresponding hole consequence of the repulsive core of the interatomic interaction. When $\rho$ increases $g(r)$ gains structure, with the main peak that shifts to shorter distances and increases its strength.

There is no experimental measurement on solid $\mathrm{H} \downarrow$ at low pressures and nothing is known experimentally about the form of its solid lattice at low temperatures. Theoretically, the solid phase of spin-polarized H (Ref. 23) was

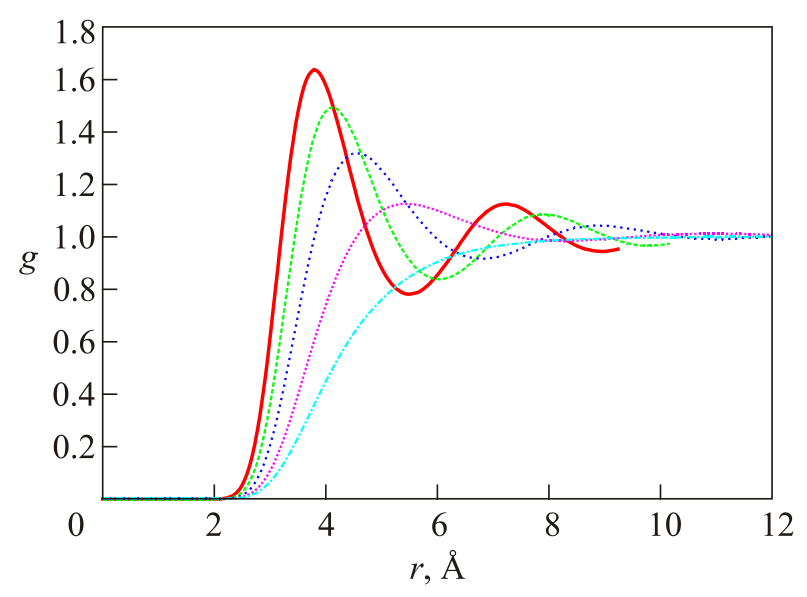

Fig. 7. (Color online) Two-body radial distribution functions of the gas phase. From bottom to top in the height of the main peak, the results correspond to densities $0.002,0.0067,0.01,0.0125$, and $0.015 \AA^{-3}$.

studied using as importance sampling trial wave function given by Eq. (4). The geometry of the lattice is defined by a proper selection of the lattice sites $\mathbf{r}_{I}$ around which the atoms are organized according to a commensurate solid. Calculations were carried out at some densities using the fcc, hcp, and bcc lattices. Near the melting density the bcc phase was slightly preferred and, at higher densities, the differences between fcc, hcp, and bcc phases were not distinguishable within the statistical noise. Therefore, bcc phase was assumed in the study of solid $\mathrm{H} \downarrow$ properties. It is worth noticing that the same lattice was used in the past by Pierleoni et al. [49] in the study of solid $\mathrm{H}$ at very high pressure.

The full set of results for the energy of the solid phase is shown in Fig. 8. The solid line on top of the DMC re-

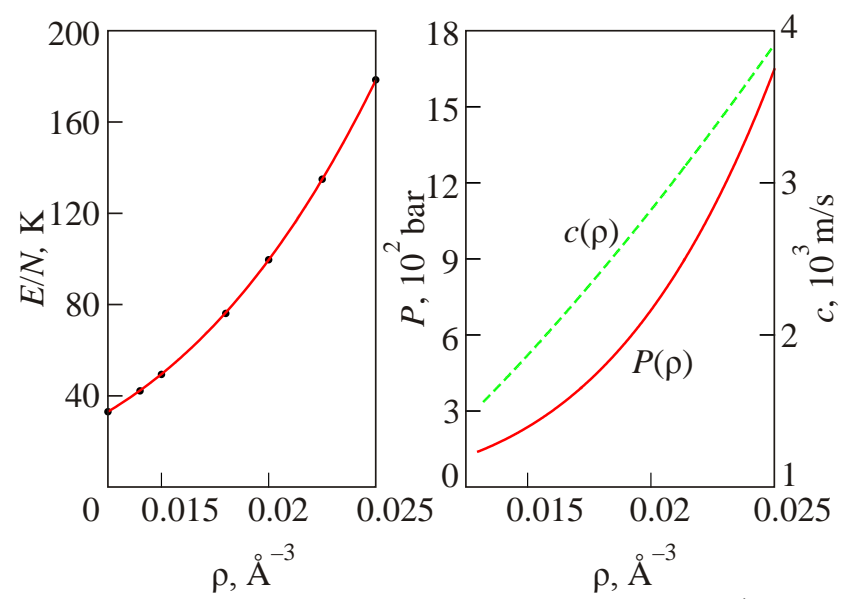

Fig. 8. (Color online) Left: Energy per particle of solid $\mathrm{H} \downarrow$ (solid circles) as a function of the density $\rho$. The solid line corresponds to the fit to the DMC energies using Eq. (16). The error bars of the DMC energies are smaller than the size of the symbols. Right: Pressure and speed of sound of solid $\mathrm{H} \downarrow$ as a function of the density. Left (right) scale corresponds to pressure (speed of sound). 
sults for the energy corresponds to a numerical fit obtained using the function

$$
e(\rho)=s_{1} \rho+s_{3} \rho^{3} .
$$

The optimal values in Eq. (16) are $s_{1}=1147(6) \mathrm{K} \cdot \AA$ and $s_{3}=9.57(2) \cdot 10^{6} \mathrm{~K} \cdot \AA^{3}$. On the same figure, at the right side are presented the results of the pressure and the speed of sound derived from the equation of state, using the same expressions as in the gas calculations.

Knowledge of the equations of state of the gas and solid phases of $\mathrm{H} \downarrow$ enabled the prediction of the location of the gas-solid phase transition, using the double-tangent Maxwell construction, as shown in Fig. 9. From the common tangent to both phases the freezing $\left(\rho_{f}\right)$ and melting $\left(\rho_{m}\right)$ densities were obtained, $\rho_{f}=0.01328 \AA^{-3}$ and $\rho_{m}=0.01379 \AA^{-3}$, which correspond to a common pressure at the transition of $P=173(15)$ bar. The melting pressure proved to be quite independent of the lattice used in the simulation since using fcc and hcp 175 and 176 bar were obtained, respectively.

This value is significantly higher than previous estimations: 50 bar, obtained by using the quantum theory of corresponding states [4], and 81 bar, from a VMC estimation by Danilowicz et al. [50]. It is worth noticing that the transition point depends dramatically on the accuracy of the theoretical method used for its calculation: estimation from the Ref. 23 using the VMC method is $P=113(17)$ bar, a value significantly smaller than the DMC result.

Other relevant quantities at the transition were determined as well. The Lindemann ratio, defined as $\gamma=\sqrt{\left\langle\left(\mathbf{r}-\mathbf{r}_{I}\right)^{2}\right\rangle} / a_{L}$, where $a_{L}$ is the lattice constant has almost the same value, $\gamma=0.25$ as in liquid ${ }^{4} \mathrm{He}$ $(\gamma=0.26)$. It was possible to determine the discontinuity in the kinetic energy at the transition and it amounts to $7.5 \mathrm{~K}$ approximately (in the gas side at freezing

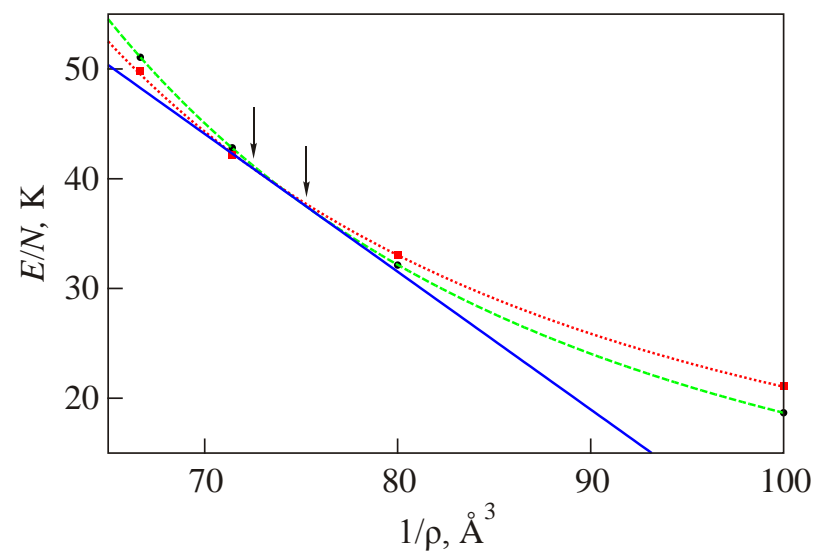

Fig. 9. (Color online) Maxwell construction based on plotting the energy per particle, $E / N$ as a function of $1 / \rho$. The densities at which the first-order transition occurs are identified by finding the common tangent (solid line) to both the solid (dotted line) and gas curve (dashed line).

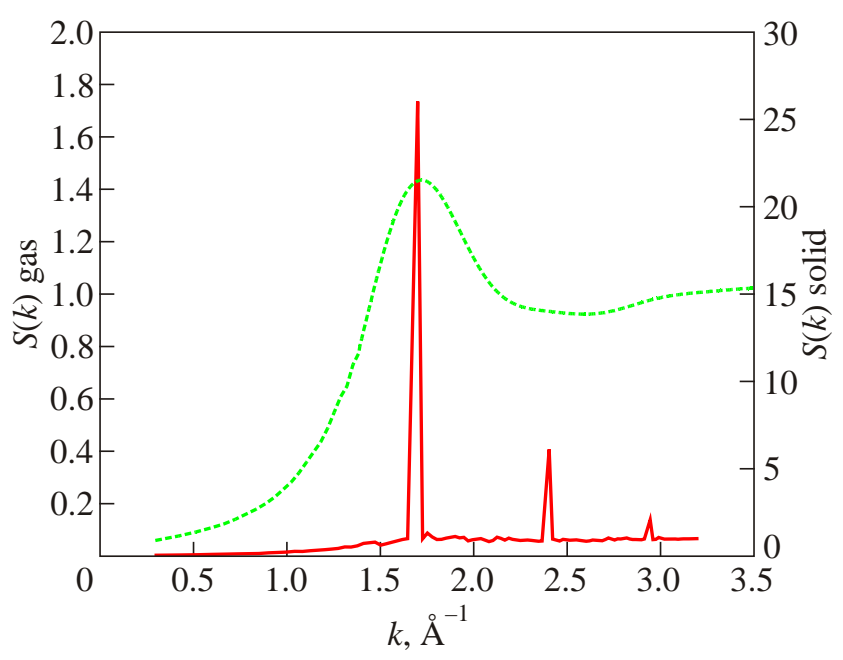

Fig. 10. (Color online) Static structure factor at the gas-solid phase transition. The results correspond to the gas at $\rho_{f}$ (dashed line) and to the solid at $\rho_{m}$ (straight line).

$T / N=44.0(5) \quad \mathrm{K}$ and in the solid side at melting $T / N=51.5(6) \mathrm{K})$. At the crystallization, the condensate fraction is small but not zero, $n_{0}=0.04$. Other structural quantities, in particular $g(r)$ and $S(k)$ were determined at the transition as well. Here, in Fig. 10, we present the comparison of $S(k)$ for both phases at densities $\rho_{f}$ (gas) and $\rho_{m}$ (solid). High intensity peaks located at the reciprocal lattice sites are a clear signature of the solid order; they are obviously absent in the $S(k)$ of the gas.

Hydrogen at finite temperature has recently been studied at very low densities using an approximate method, the static fluctuation approximation [51]. The condensate fraction, transition temperature and specific heat per particle have been determined. In accordance with the results of the dilute bosonic systems, it has been shown that the transition temperature is greater than that for the noninteracting gas.

\section{2. $\mathrm{H} \downarrow$ in $2 D$}

Spin-polarized hydrogen in 2D is an example of a Bose system with reduced dimensionality, with an additional interest of well known interaction between particles. Part of the interest in its properties comes from the fact that it can be considered as a possible model for hydrogen adsorbed on the surface of liquid helium, where a quasicondensate has been observed. Recently, $\mathrm{H} \downarrow$ in 2D has been studied at $T=0$ by the DMC method [26]. Similarly to the situation in 3D, at very low densities both the energy and the condensate fraction of gas $\mathrm{H} \downarrow$ show universal behavior in terms of the gas parameter $\sigma a^{2}$. This can be seen in Fig. 11 from the comparison of the $\mathrm{H} \downarrow$ results [26] with the hard disk energies per particle in 2D calculated by Pilati et al. [52] and the mean-field results given by

$$
\frac{E_{M F}}{N}=\frac{2 \pi \hbar^{2}}{m} \frac{1}{\ln \left(1 / \sigma a^{2}\right)} .
$$



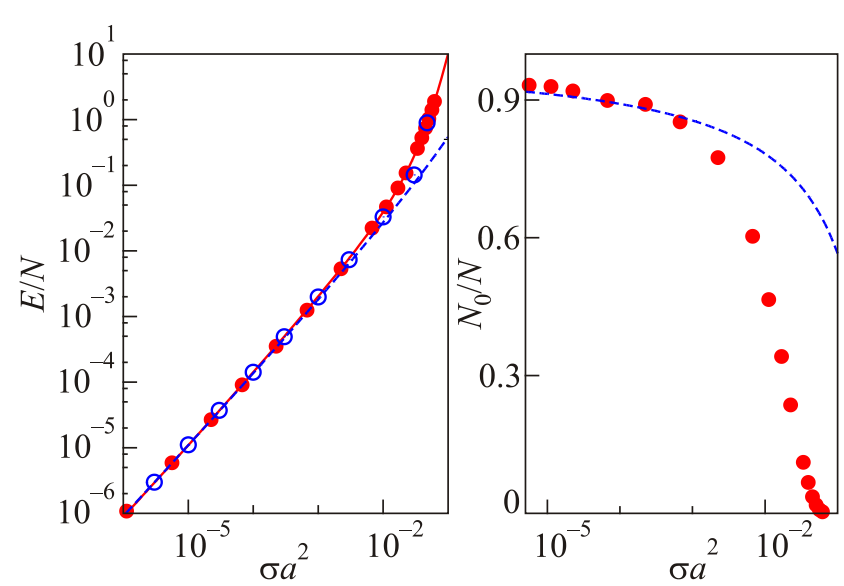

Fig. 11. (Color online) Left: Equation of state of the gas $\mathrm{H} \downarrow$ as a function of the gas parameter $\sigma a^{2}$ in units $\hbar^{2} /\left(2 m a^{2}\right)$ (solid circles and full line). The empty circles correspond to the HS gas [52] and the line to the mean-field results (Eq. 17). Right: Condensate fraction as a function of the gas parameter (circles). Dashed line is the result from the Bogoliubov theory.

As previously observed in a 3D gas, the energies per particle of $\mathrm{H} \downarrow$ start to deviate from the mean-field results sooner than in the case of hard spheres. In addition, the corrections to the mean-field results in 2D can not be well fitted with the functional form appropriate for the hard sphere gas [52].

Similar behavior is observed for the condensate fraction, where for higher densities the Bogoliubov formula [53]

$$
\frac{N_{0}}{N}=1+\frac{1}{\ln \left(\sigma a^{2}\right)},
$$

overestimates the condensate fraction, which was previously observed also for the hard disks [52]. In comparison with the 3D $\mathrm{H} \downarrow$ gas condensate depletion is larger in a 2D gas.

At higher densities a phase transition to a triangular solid phase occurs. The equation of state, as well as the pressure and the speed of sound derived from it are presented in Fig. 12. From the equations of state of gas and solid,
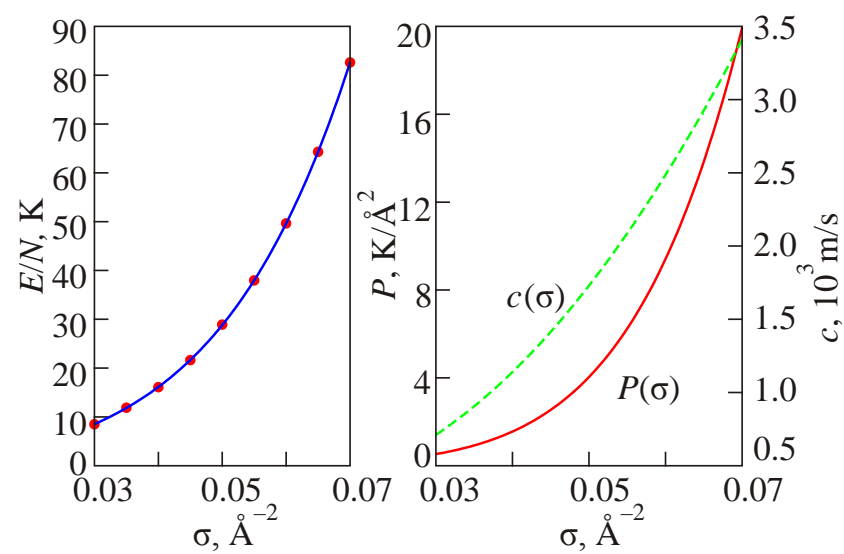

Fig. 12. (Color online) Left: Energy per particle of solid $\mathrm{H} \downarrow$ as a function of the density. Right: Pressure and speed of sound of solid $\mathrm{H} \downarrow$ as a function of the density. Left-(right-) hand scale corresponds to pressure (speed of sound). using double-tangent Maxwell construction it was predicted that gas-solid phase transition occurs at the freezing $\sigma_{f}=0.0407 \AA^{-2}$ and melting $\sigma_{m}=0.0417 \AA^{-2}$ density. They correspond to a common pressure at the transition of $P=1.84 \mathrm{~K} / \AA^{2}$.

Recently, the static fluctuation approximation was used to study finite $2 \mathrm{D} H \downarrow$ in a very low-density regime at finite temperature [54]. The results on the condensate fraction and specific heat capacity show that the BEC occurs in the system.

\subsection{Spin-polarized hydrogen adsorbed on the surface of liquid helium}

The experimental realization of a thin layer of spinpolarized hydrogen $H \downarrow$ adsorbed on top of the surface of superfluid ${ }^{4}$ He provides one of the best examples of a stable nearly two-dimensional quantum Bose gas [8]. Theoretically, in a pioneering work, Mantz and Edwards [55] used the variational Feynman-Lekner approximation to calculate the effective potential felt by a hydrogen atom on the ${ }^{4}$ He surface. Solving the Schrödinger equation for the atom in this effective potential they concluded that $\mathrm{H} \downarrow$, $\mathrm{D} \downarrow$, and $\mathrm{T} \downarrow$ have a single bound state and calculated the respective binding energies. The main drawback of this treatment is that the adsorbent is substituted by an effective field representing a static and undisturbed surface. In fact, a quantitatively accurate approach to this problem requires of a good model for the ${ }^{4}$ He surface. Recently, the system was studied using accurate interparticle potentials and quantum Monte Carlo methods in the zero-temperature limit [27]. In order to guarantee an accurate model for the free surface of ${ }^{4} \mathrm{He}$, a slab geometry was used, based on experience in the previous study of the free ${ }^{4} \mathrm{He}$ surface [56]. The resulting density profile of the system under study is presented in Fig. 13. $\mathrm{H} \downarrow$ layer has an approximate width of $8 \AA$ and virtually floats on the helium surface: the center of the $\mathrm{H} \downarrow$ layer is located out of the surface, where the ${ }^{4} \mathrm{He}$ density is extremely small. Similar picture was obtained by Mantz and Edwards [55] in a variational description of the adsorption of a single $\mathrm{H} \downarrow$ atom. It also resemb-

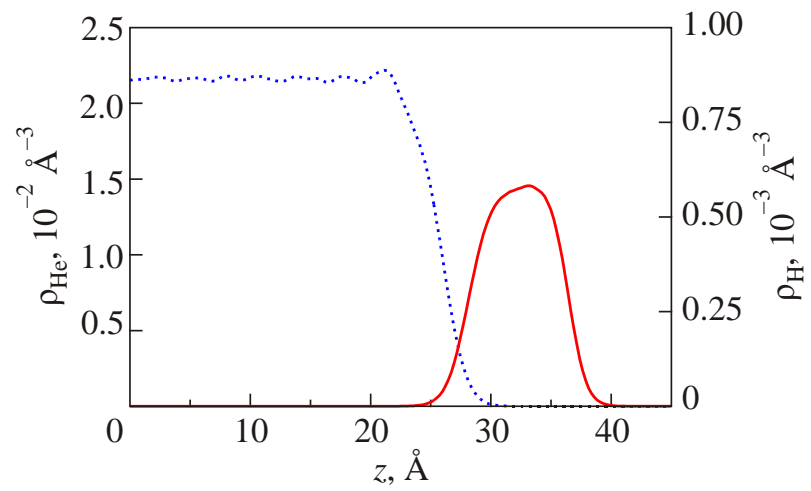

Fig. 13. (Color online) Density profile of the ${ }^{4} \mathrm{He}$ slab (dashed line) and of the $H \downarrow$ adsorbed gas (solid line) corresponding to a surface density $\sigma=9.57 \cdot 10^{-3} \AA^{-2}$. 
les the case of ${ }^{3} \mathrm{He}$ adsorbed on the ${ }^{4} \mathrm{He}$ surface [57], however in that case ${ }^{3} \mathrm{He}$ distribution is centered not so far from the bulk.

The obtained energies per particle at different coverages were compared with the 2D simulation in order to establish the degree of two-dimensionality of the adsorbed gas. The comparison presented in Fig. 14 shows the agreement between the strictly 2D gas and the film is good for densities $\sigma \leq 5 \cdot 10^{-3} \AA^{-2}$. At higher densities, the additional degree of freedom in the $z$ direction results in nearly linear growth of the energy with the surface density in the layer up to the shown density, in contrast with the significant quadratic increase observed in the 2D gas $\left(C<<C_{2 D}\right)$. The lines on top of the data represent polynomial fits to the DMC energies of the gas. For $\mathrm{H} \downarrow$ adsorbed on the surface of ${ }^{4} \mathrm{He}$,

$$
E / N(\sigma)=B \sigma+C \sigma^{2},
$$

with optimal parameters $B=48(2) \quad K \cdot \AA^{2}$ and $C=5.6(9) \cdot 10^{2} \mathrm{~K} \cdot \AA^{4}$, the figures in parenthesis being the statistical uncertainties. Before performing the fit, the energy of a single $\mathrm{H} \downarrow$ atom, or in other words, the energy in the infinite dilution limit $\sigma \rightarrow 0$, was subtracted from the computed energies enabling thus better visualization of the results and comparison to $2 \mathrm{D}$ calculation. The results obtained for the energy per particle of the 2D gas are well reproduced by a polynomial low

$$
E / N(\sigma)=B_{2 D} \sigma+C_{2 D} \sigma^{2},
$$

with $B_{2 D}=35(3) \mathrm{K} \cdot \AA^{2}$ and $C_{2 D}=6.4(1) \cdot 10^{4} \mathrm{~K} \cdot \AA^{4}$.

Since the energy of the layer departs from the twodimensional behavior with the increase of the density, it was investigated if the existence of a nearly 3D gas is possible. Using several estimates for the width of the layer and

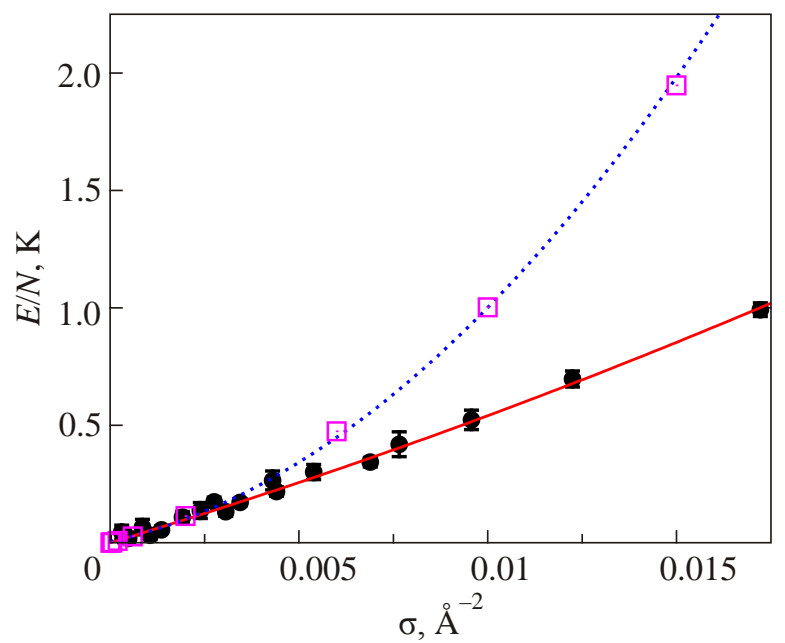

Fig. 14. (Color online) Comparison between the energy per particle of $\mathrm{H} \downarrow$ adsorbed on the ${ }^{4} \mathrm{He}$ slab (full circles) and the energy of purely two-dimensional $\mathrm{H} \downarrow$ (open squares). The solid line is the polynomial fit (19) and the dashed line is a fit on the 2D energies (20).

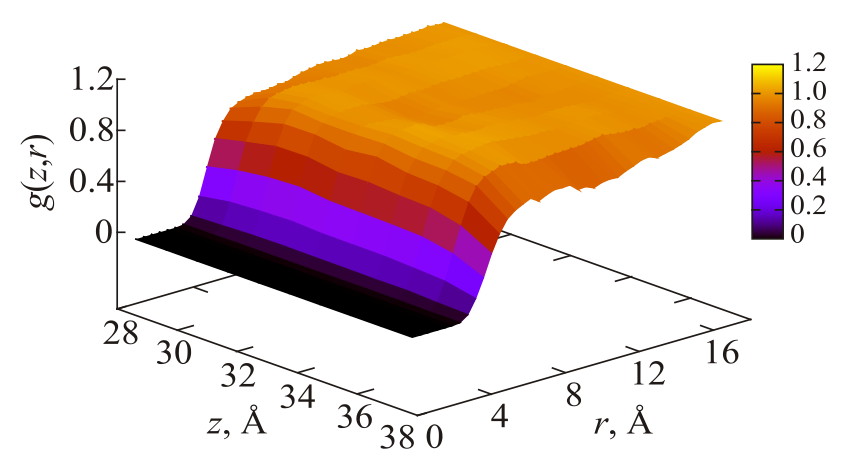

Fig. 15. (Color online) Two-body distribution function $g(z, r)$ of $\mathrm{H} \downarrow$ adsorbed on ${ }^{4} \mathrm{He}$, with $r=\sqrt{x^{2}+y^{2}}$, at surface density $\sigma=0.0215 \AA^{-2}$.

comparing the results with the energy of the 3D gas it was shown that the energies of adsorbed $\mathrm{H} \downarrow$ are not well described by a 3D equation of state at any density within the regime studied. Structural quantities of interest were determined as well and compared with 2D distributions. The distribution functions of $\mathrm{H} \downarrow$ atoms in the layer were studied by doing slices of small width ( $\Delta z=1 \AA)$ and, within a given slice, as a function of the radial distance in the plane $r=\sqrt{x^{2}+y^{2}}$. Results of the two-body radial distribution function $g(z, r)$ where $z$ is the distance to the center of the ${ }^{4} \mathrm{He}$ slab at a coverage $\sigma=0.0215 \AA^{-2}$ are presented in Fig. 15. Around the center of the $\mathrm{H} \downarrow$ density profile, $g(r)$ is nearly independent of $z$ with a main peak of a height smaller than 1.2. In the wings of $\rho_{\mathrm{H}}(z)$, where the local density is smaller, $g(r)$ shows less structure and the noise of the DMC data also increases due to low statistics.

Other particularly relevant magnitude is the one-body distribution function $\rho_{1}(r)$ since it furnishes evidence of the presence of off-diagonal long-range order in the system. It was studied in the same way as the two-body radial distribution function.

In order to study the dimensionality of the off-diagonal long-range order in the adsorbed gas the comparison was made between $\rho_{1}(r)$ for a 2D gas and for a slice in the centre of the adsorbed layer at the same density. Additionally, from the asymptotic behavior of the one-body density matrix in the central part of the density profile the condensate fraction $n_{0}$ was determined. The results presented in Fig. 16 show that the behavior of $\mathrm{H} \downarrow$ in the layer is significantly different from the one observed in strictly 2D. The difference is larger than the one observed at the same density for $g(r)$, with values for the condensate fraction, that differ in a $\sim 30 \%$. Larger value of the condensate fraction in the layer occurs because atoms have the transverse degree of freedom $z$ that translates into an effective surface density smaller than the one of the 2D layer.

The comparison of the condensate fractions in the layer and in 2D as a function of coverage is show in Fig. 17. As expected, the condensate fraction in the layer is nearly 1 at very low densities and then decreases when $\sigma$ increases. 


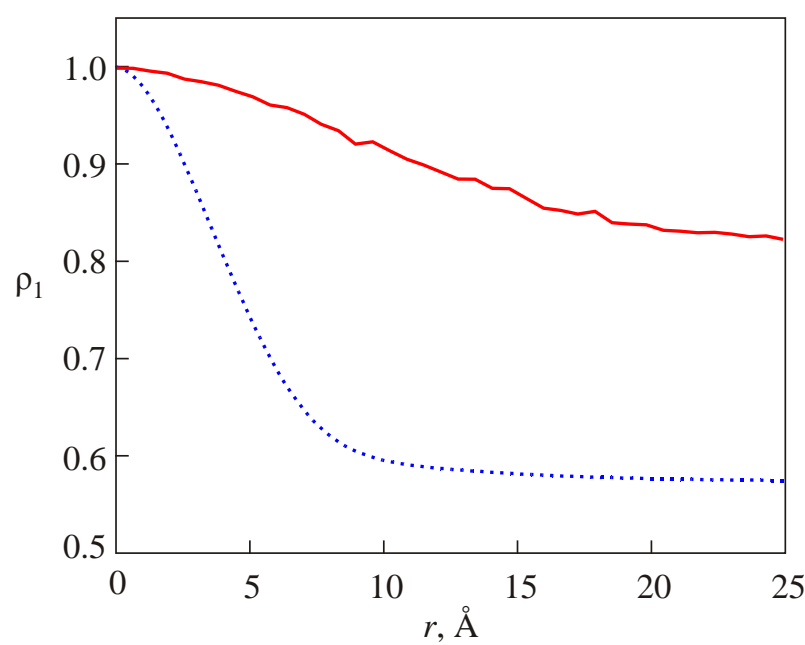

Fig. 16. (Color online) Comparison between the one-body distribution function in the centre of the slab, corresponding to a density $\sigma=0.0095 \AA^{-2}$ (solid line) with the one corresponding to a purely 2D $\mathrm{H} \downarrow$ gas at the same surface density (dotted line).

However, the decrease is quite slow in such a way that even at densities as large as $\sigma=0.02 \AA^{-2}$ the condensate fraction is still $n_{0} \simeq 0.6$. At the same density, the condensate fraction of the $2 \mathrm{D}$ gas is half this value, $n_{0} \simeq 0.3$. The dependence of $n_{0}$ with the density for the 2D geometry, is significantly stronger with a larger depletion of the condensate fraction for all densities.

\section{Spin-polarized deuterium}

Quantum Monte Carlo methods were employed recently in the study of the three spin-polarized deuterium species, $\mathrm{D} \downarrow_{1}, \mathrm{D} \downarrow_{2}$ and $\mathrm{D} \downarrow_{3}$ at zero temperature [25,29]. In Fig. 18, the DMC energies per particle calculated with a guiding wave function including backflow correlations, are plott-

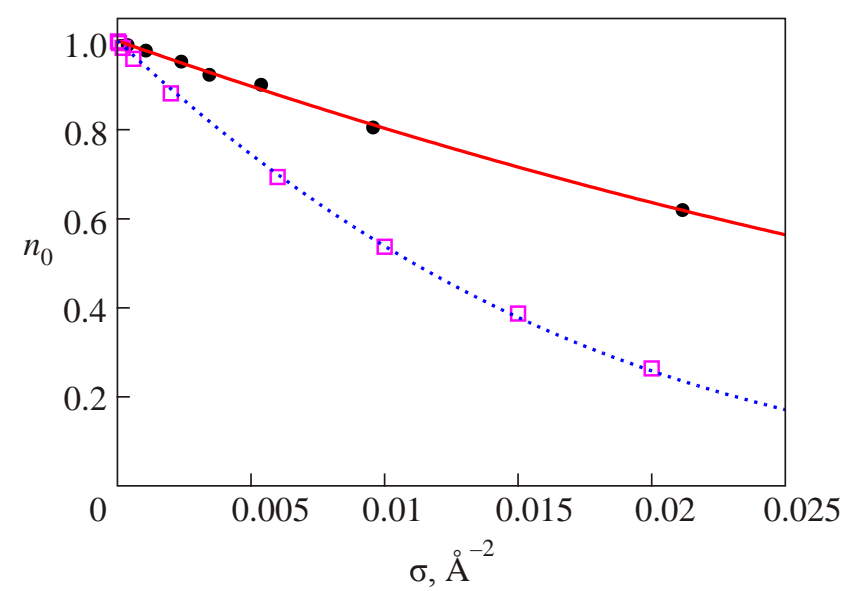

Fig. 17. (Color online) Condensate fraction as a function of the surface density $\sigma$. Solid circles correspond to $\mathrm{H} \downarrow$ on ${ }^{4} \mathrm{He}$ and open squares to a $2 \mathrm{D}$ gas. The lines on top of the DMC data are fits to guide the eye.

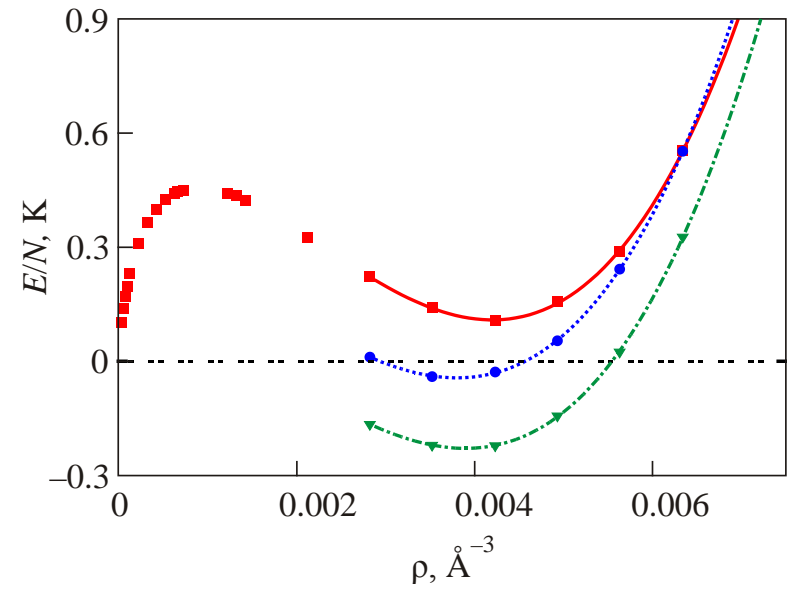

Fig. 18. (Color online) Energy per particle of $\mathrm{D} \downarrow_{1}$ (squares), $\mathrm{D} \downarrow_{2}$ (circles) and $\mathrm{D} \downarrow_{3}$ (triangles) as a function of the density $\rho$, with backflow correlations included in the model. The lines correspond to fits to the DMC energies using Eq. (21). The error bars of the DMC energies are smaller than the size of the symbols.

ed as a function of the density for $\mathrm{D} \downarrow_{1}, \mathrm{D} \downarrow_{2}$ and $\mathrm{D} \downarrow_{3}$. The lines reported in the same figure are the equations of state obtained with a numerical fit to the DMC data using a polynomial form (21). The DMC results were fitted in the density range from 0.00282 to $0.00634 \AA^{-3}$ and the best set of fitting parameters are reported in Table 2 . As it was expected due to the degeneracy, the precise numerical values from Table 2 indicate that the energy ordering for the three $\mathrm{D} \downarrow$ species, close to the equilibrium densities, is $(E / N)_{D \downarrow_{1}}>(E / N)_{D \downarrow_{2}}>(E / N)_{D \downarrow_{3}}$. It is also obvious that with the increase of degeneracy, the equilibrium density $\rho_{0}$ shifts to slightly smaller values.

The obtained negative energy per particle at zero pressure, i.e., at minimum of the equation of state of $\mathrm{D} \downarrow_{2}$ and $\mathrm{D} \downarrow_{3}$, confirms their liquid ground state (Table 2). A small positive energy per particle at zero pressure, i.e., minimum in the equation of state of $\mathrm{D} \downarrow_{1}$, does not allow to qualify its ground state as a liquid (Table 2). In fact, due to this peculiarity in the $D \downarrow_{1}$ equation of state, it was pointed out in Ref. 58 that $\mathrm{D} \downarrow_{1}$ could be the case of most experimental interest. Namely, the authors concluded that $\mathrm{D} \downarrow_{1}$ may remain in the gaseous state down to absolute zero, leaving open the possibility that $\mathrm{D} \downarrow_{1}$ could liquefy under a very slight pressure. The liquid-gas coexistence region in $\mathrm{D} \downarrow_{1}$ was also predicted in previous variational calculations [19].

Table 2. Parameters of the equation of state (21) of $\mathrm{D} \downarrow_{1}$, D $\downarrow_{2}$ and $\mathrm{D} \downarrow_{3}$

\begin{tabular}{l|c|c|c}
\hline \hline Parameter & $\mathrm{D} \downarrow_{1}$ & $\mathrm{D} \downarrow_{2}$ & $\mathrm{D} \downarrow_{3}$ \\
\hline \hline$e_{0}, \mathrm{~K}$ & $0.1086(8)$ & $-0.043(2)$ & $-0.181(2)$ \\
$B, \mathrm{~K}$ & $1.31(2)$ & $0.96(2)$ & $0.87(4)$ \\
$C, \mathrm{~K}$ & $0.8(1)$ & $0.56(6)$ & $0.52(7)$ \\
$\rho_{0}, \AA^{-3}$ & $0.00420(3)$ & $0.00381(5)$ & $0.00372(2)$ \\
\hline \hline
\end{tabular}


In order to resolve the theoretical puzzle concerning the liquid-gas coexistence region in $\mathrm{D} \downarrow_{1}$, a double-tangent Maxwell construction was used. The method required the use of the universal expansion of the equation of state of a Fermi gas in the region of very small densities [59]. VMC results predicted a first order gas-liquid transition at the gas density $\rho=5.4 \cdot 10^{-5} \AA^{-3}$ (liquid density $\rho=$ $=0.00398(1) \AA^{-3}$ ) and pressure $p \sim 8 \cdot 10^{-4}$ bar. According to the DMC results, a first order gas-liquid transition occurs even at a smaller gas density, $\rho=1.48 \cdot 10^{-5} \AA^{-3}$ (liquid density $\rho=0.00421(1) \AA^{-3}$ ), and at lower pressure, $\quad p \sim 9 \cdot 10^{-5}$ bar. It that way, both results confirm the previous theoretical prediction reported in Ref. 19.

The implementation of the best nowadays available tool for microscopic studies of Fermi systems, the diffusion Monte Carlo method within the fixed-node approximation, resulted with the most accurate description of the macroscopic character of the three $\mathrm{D} \downarrow$ species. The obtained $\mathrm{D} \downarrow_{1}, \quad \mathrm{D} \downarrow_{2}$ and $\mathrm{D} \downarrow_{3}$ equations of state allowed for determination of the pressure and the speed of sound as a function of the density of the system (Eqs. (14) and (15)). For a weakly self-bound liquids $\mathrm{D} \downarrow_{2}$ and $\mathrm{D} \downarrow_{3}$ the functions $P(\rho)$ and $c(\rho)$ are reported in Fig. 19, and from the presented results it is clear that the pressure and the speed of sound in $\mathrm{D} \downarrow_{2}$ and $\mathrm{D} \downarrow_{3}$ liquids assume similar values. In addition, the densities at which those liquids become unstable with respect to density fluctuations were localized. The results show that in liquid $\mathrm{D} \downarrow_{2}$ the speed of sound becomes zero at the density $\rho_{s}=0.002813 \AA^{-3}$, and at a very small negative pressure $P_{S}=-0.11(1)$ bar. As it is shown in Fig. 19, in liquid $\mathrm{D} \downarrow_{3}$ the speed of sound becomes zero at slightly higher density, $\rho_{s}=0.002903 \AA^{-3}$, while the spinodal pressure $P_{S}=-0.12(1)$ bar is similar to liquid $\mathrm{D} \downarrow_{2}$ spinodal pressure.

Since $\mathrm{D} \downarrow_{3}$ is a three-component degenerate Fermi liquid it does not posses its helium analogue, but $\mathrm{D} \downarrow_{2}$ with

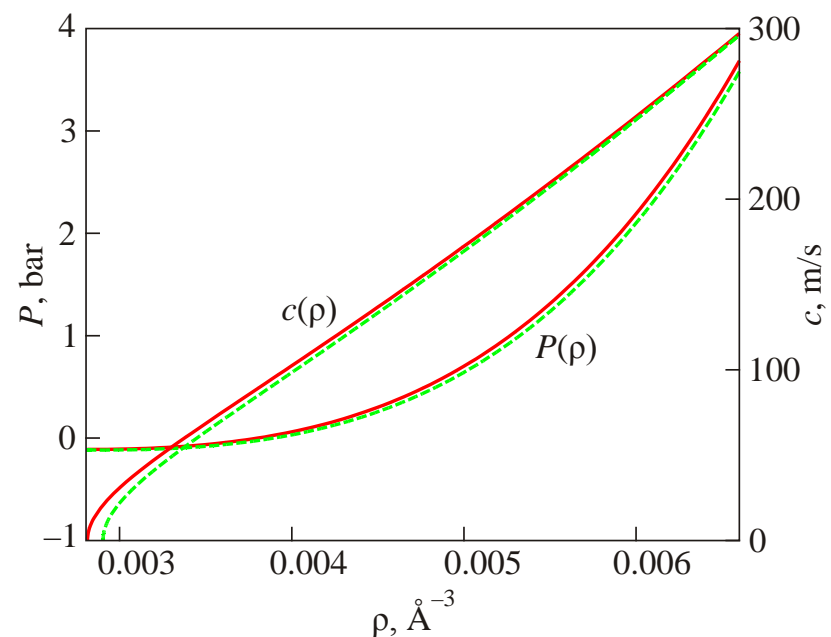

Fig. 19. (Color online) Pressure and speed of sound of $\mathrm{D} \downarrow_{2}$ (solid lines) and $\mathrm{D} \downarrow_{3}$ (dashed lines) as a function of the density. Left (right) scale corresponds to pressure (speed of sound).
Table 3. Comparison of the main relevant properties of liquids $\mathrm{D} \downarrow_{2}$ and ${ }^{3} \mathrm{He}$. The corresponding $\sigma \quad\left(\sigma_{\mathrm{H}}=3.67 \AA\right.$ and $\sigma_{\mathrm{He}}=2.556 \AA$ ) are used for each liquid. The results for ${ }^{3} \mathrm{He}$ are taken from Ref. 33

\begin{tabular}{l|c|c}
\hline \hline Parameter & $\mathrm{D}_{2}$ & ${ }^{3} \mathrm{He}$ \\
\hline \hline$\rho_{0}, \sigma^{-3}$ & 0.188 & 0.274 \\
$e_{0}, \mathrm{~K}$ & -0.043 & -2.464 \\
$\rho_{s}, \sigma^{-3}$ & 0.139 & 0.202 \\
$P_{S}$, bar & -0.11 & -3.09 \\
\hline \hline
\end{tabular}

degeneracy two has its helium analogue in unpolarized liquid ${ }^{3} \mathrm{He}$. It is useful to compare the representative quantities of $\mathrm{D} \downarrow_{2}$ and ${ }^{3}$ He liquids in units of $\sigma$, and in order to that we summarized in Table 3 the main relevant properties of those liquids. One can notice a significant difference between the parameters that describe the nature of the ground states of $\mathrm{D} \downarrow_{2}$ and ${ }^{3} \mathrm{He}$ liquids. As in the comparison of $\mathrm{T} \downarrow$ and ${ }^{4}$ He liquids, a part of the difference can be due to the mass difference and the difference between the interaction potentials, but in the comparison between $\mathrm{D} \downarrow_{2}$ and ${ }^{3}$ He there is another additional source that is causing a difference, and that is their Fermi nature. An effective way to emphasize this difference even more is to observe the spatial structure of the ground state of both liquids. To do that, we plotted in Fig. 20 the two-body radial distribution functions $g(r)$ at the equilibrium densities of ${ }^{3} \mathrm{He}$ and $\mathrm{D} \downarrow_{2}$ liquids, expressing $r$ in terms of corresponding $\sigma$. The stronger interaction between ${ }^{3} \mathrm{He}$ atoms can be recognized through a higher main peak and through an evident formation of the second peak.

The description of the three $\mathrm{D} \downarrow$ species from the structural point of view can be realized by analyzing the profiles of the two-body radial distribution function $g(r)$ and static structure function $S(k)$. Since the general behavior of those functions is similar for all three $\mathrm{D} \downarrow$ species, we

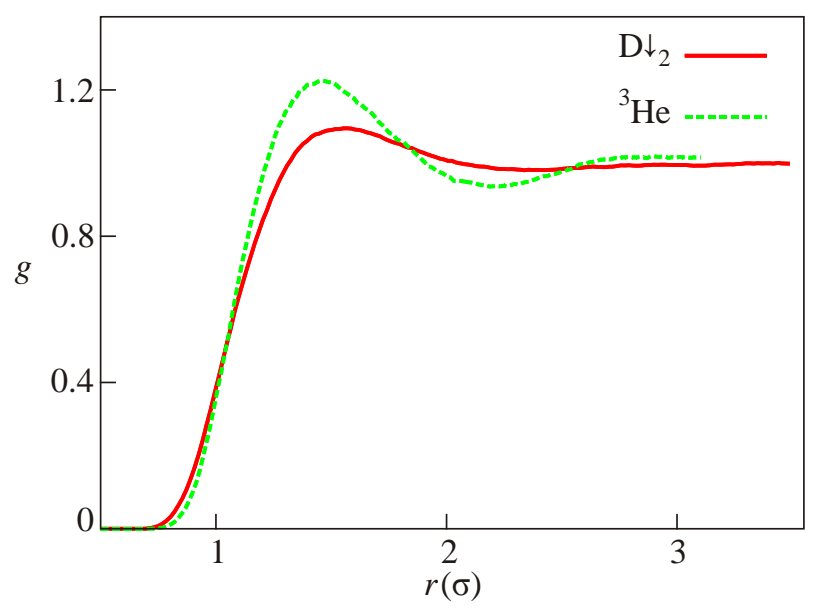

Fig. 20. (Color online) Two-body radial distribution functions of ${ }^{3} \mathrm{He}\left(r\right.$ in $\left.\sigma_{\mathrm{He}}\right)$ and $\mathrm{D} \downarrow_{2}\left(r\right.$ in $\left.\sigma_{\mathrm{H}}\right)$ liquids at the equilibrium densities. 


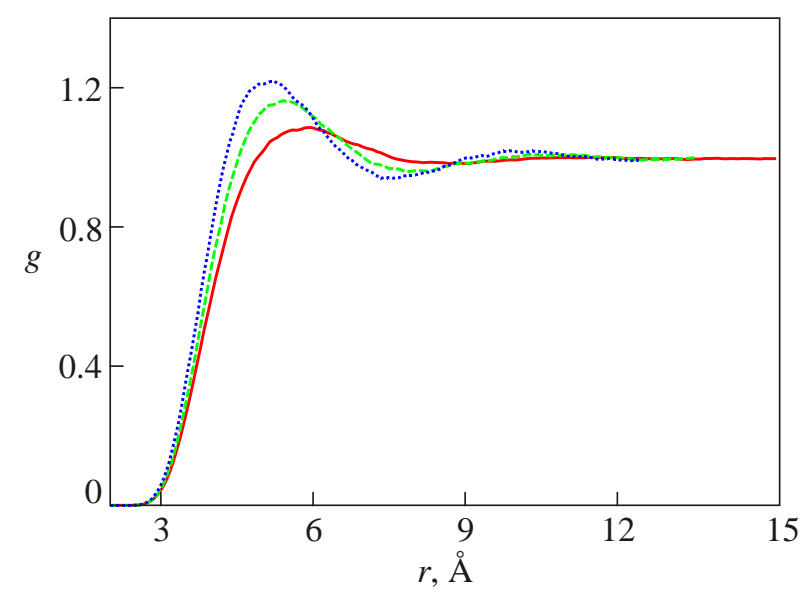

Fig. 21. (Color online) Two-body radial distribution function of $\mathrm{D} \downarrow_{3}$. From bottom to top in the height of the main peak, the results correspond to densities $0.00352 \AA^{-3}$ (solid line), $0.00493 \AA^{-3}$ (dashed line), and $0.00634 \AA^{-3}$ (dotted line).

present it using the example of liquid $D \downarrow_{3}$ because this three-component Fermi liquid does not have its helium analogue, and in that sense represents completely novel system. For three different densities the DMC results for $g(r)$ and $S(k)$ are plotted in Figs. 21 and 22, obtained using the method of pure estimators [32]. As it is shown in Fig. 21, a very well known behavior for $g(r)$ is also present in the three-component Fermi liquid $D \downarrow_{3}$. The shift of the main peak to shorter distances is clearly observed when the density increases, as well as the growth of its strength. The increase of the density enforces the formation of the more pronounced structure in the system, that can be recognized through the appearance of the secondary peaks. The reported results for $S(k)$ in Fig. 22 are the Fourier transforms of the $g(r)$ functions, except in the region of very small $k$ where the results are obtained directly from the DMC calculations. As can be seen, a familiar

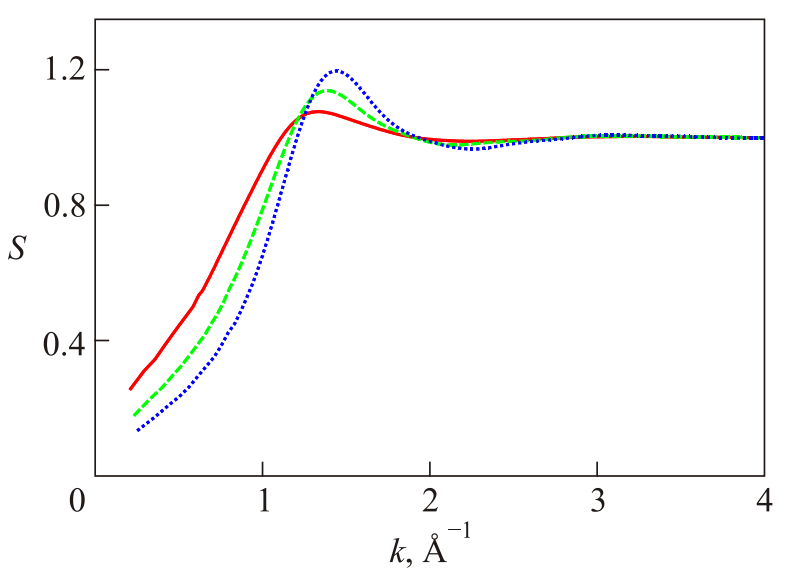

Fig. 22. (Color online) Static structure function of $\mathrm{D} \downarrow_{3}$. From bottom to top in the height of the main peak, the results correspond to densities $0.00352 \AA^{-3}$ (solid line), $0.00493 \AA^{-3}$ (dashed line), and $0.00634 \AA^{-3}$ (dotted line). behavior for $S(k)$ in liquid is shown, i.e., the strength of the main peak increases and moves to higher moment with the increase of the density.

In addition to the previously reported results for the total $g(r)$ of $\mathrm{D}_{3}$, the two-body radial distribution function for atoms having the same spin orientation in $D \downarrow_{1}, D \downarrow_{2}$ and $\mathrm{D} \downarrow_{3}$ were also studied, and the two-body radial distribution function for atoms having different spin orientation in $\mathrm{D} \downarrow_{2}$ and $\mathrm{D} \downarrow_{3}$. Again, we decided to use liquid $\mathrm{D} \downarrow_{3}$ to describe one property which is direct consequence of the Fermi nature of the system. By comparing the results presented in Fig. 23, for atoms having the same and different spin orientation one can notice different behaviors. In Fig. 23(a), the formation of the main peak is practically visible just at the highest density shown $\rho=0.00634 \AA^{-3}$, while in Fig. 23(b) the main peak is clearly visible at all included densities. In addition, the main peak at the density $\rho=0.00634 \AA^{-3}$ in Fig. 23(b) is significantly higher $\left(g\left(r_{m}\right) \simeq 1.36\right)$ than any value of $g(r)$ at the same density in Fig. 23(a), where $g(r)<1.05$. The difference between the spin dependent two-body radial distribution profiles can be explained with Pauli principle. Namely, in the case
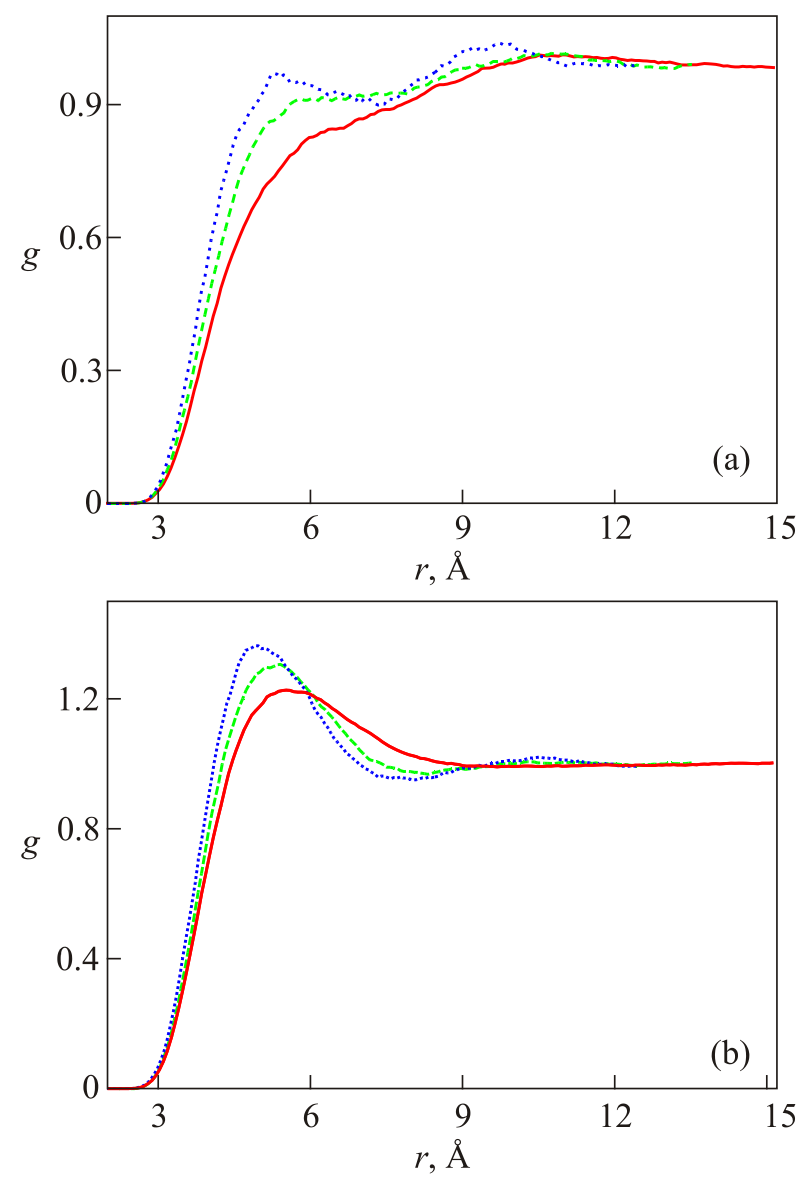

Fig. 23. (Color online) Two-body radial distribution functions of liquid $\mathrm{D} \downarrow_{3}$ for atoms having the same (a) and different (b) spin orientations. From bottom to top in the height of the main peak, the results correspond to densities $0.00352 \AA^{-3}$ (solid line), $0.00493 \AA^{-3}$ (dashed line), and $0.00634 \AA^{-3}$ (dotted line). 


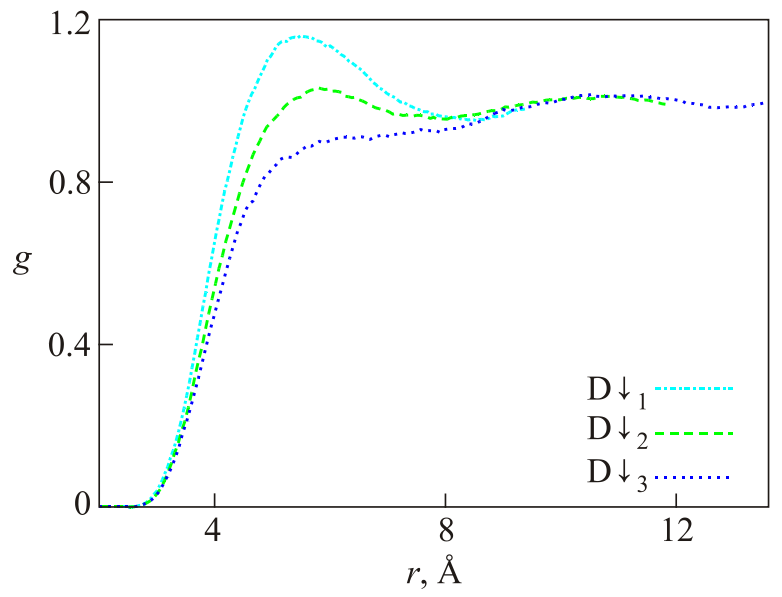

Fig. 24. (Color online) Two-body radial distribution function for $\mathrm{D} \downarrow_{1}, \mathrm{D} \downarrow_{2}$ and $\mathrm{D} \downarrow_{3}$ at the density $0.00493 \AA^{-3}$ for atoms having the same spin orientations.

of atoms having the same spin orientation the repulsion is more effective and because of that atoms prefer to reside at larger interparticle distances. As a consequence, the formation of the main peak at small $r$ is practically absent in Fig. 23(a) at smaller densities, and at the highest shown density $\rho=0.00634 \AA^{-3}$ the secondary peak is more pronounced than the first peak. Contrarily, due to the effective attraction between atoms having different spin orientations, the atoms prefer to reside at smaller interparticle distances, and because of that the main peak is clearly observed at all densities in Fig. 23(b).

We present the spin dependent $g(r)$ at the density $0.00493 \AA^{-3}$ in Figs. 24 and 25 to display correlations between the spin dependent $g(r)$ and the level of the degeneracy. It is interesting to notice that in case of $g(r)$ of atoms having the same spin orientation reported in Fig. 24, the increase of the degeneration produces the effect that can be addressed as the "density reduction". Obviously, the

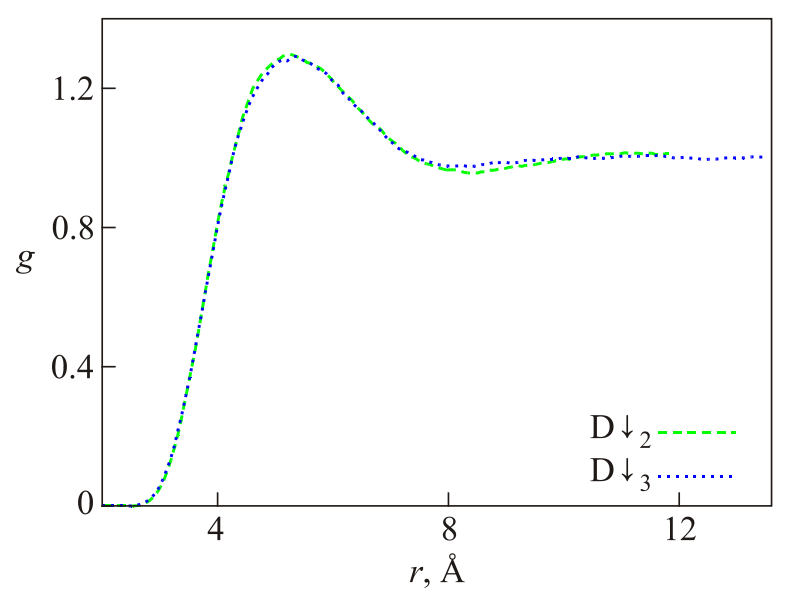

Fig. 25. (Color online) Two-body radial distribution function for $\mathrm{D} \downarrow_{2}$ and $\mathrm{D} \downarrow_{3}$ at the density $0.00493 \AA^{-3}$ for atoms having different spin orientation. product of the Slater determinants in $\mathrm{D} \downarrow_{2}$ and $\mathrm{D} \downarrow_{3}$ allows for the atoms having the same spin orientation to reside at larger interparticle distances, in the way reducing effectively the repulsion between them. This effect is not present in case of $g(r)$ of atoms having different spin orientation, as it is shown in Fig. 25. One can see that the heights of the reported peaks are almost the same, as well as the distances at which the main peaks occur in $D \downarrow_{2}$ and $\mathrm{D} \downarrow_{3}$ for atoms having different spin orientation. This can be taken as a proof that the increase of the degeneration does not effect the interparticle distances of the atoms having different spin orientations. Having this in mind, we conclude that a reduction of the repulsion between the atoms having the same spin orientation is important in lowering of the equilibrium energy per particle $e_{0}$ in $\mathrm{D} \downarrow_{2}$ and $\mathrm{D} \downarrow_{3}$ liquids, as well as for the shift of the equilibrium density $\rho_{0}$ to a slightly smaller value (Table 2 ).

\section{Spin-polarized tritium}

\section{1. $\mathrm{T} \downarrow$ in $3 D$}

The ground-state properties of spin-polarized tritium $\mathrm{T} \downarrow$ were investigated recently with quadratic DMC and the very precise triplet pair potential $b^{3} \Sigma_{u}^{+}[24,28]$. The liquid phase was studied in the density interval between the spinodal and freezing densities. In Fig. 26, the DMC energies per particle are plotted as a function of the density. The line shown in the same figure corresponds to the numerical fit to the DMC data using a polynomial function

$$
e(\rho)=e_{0}+B\left(\frac{\rho-\rho_{0}}{\rho_{0}}\right)^{2}+C\left(\frac{\rho-\rho_{0}}{\rho_{0}}\right)^{3} .
$$

In the given expression (21) the energy per particle is denoted as $e(\rho)$, the equilibrium density as $\rho_{0}$, and the energy per particle at equilibrium density as $e_{0}$. The best re-

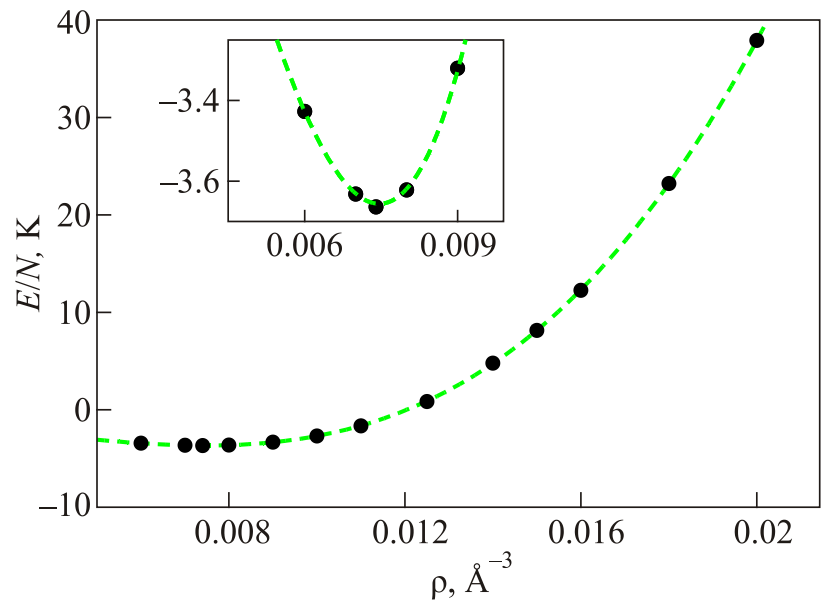

Fig. 26. (Color online) Energy per particle of liquid $\mathrm{T} \downarrow$ (solid circles) as a function of the density $\rho$. The dashed line corresponds to the fit to the DMC energies using Eq. (21). The error bars of the DMC energies are smaller than the size of the symbols. 
sulting fitting parameters were: $e_{0}=-3.656(4) \mathrm{K}$, $B=6.86(7) \mathrm{K}, C=4.70(5) \mathrm{K}$, and $\rho_{0}=0.007466(7) \AA^{-3}$, where the figures in parenthesis are the statistical uncertainties. The inset shown in Fig. 26 gives better insight in to the minimum of the equation of state of the liquid phase, i.e., to the results in the vicinity of $\rho_{0}$.

Since both, spin-polarized tritium atoms as well as ${ }^{4} \mathrm{He}$ atoms obey the Bose statistics, it is useful to compare the main properties of liquids $\mathrm{T} \downarrow$ and ${ }^{4} \mathrm{He}$, and in order to that we report in Table 4 relevant properties for both liquids. For liquid $\mathrm{T} \downarrow$, the equilibrium density expressed in units of $\sigma$ is $\rho_{0}=0.369 \sigma^{-3}\left(\sigma_{H}=3.67 \AA\right.$ ), and is very similar to the one of liquid ${ }^{4} \mathrm{He}$ given in Ref. 33, $\rho_{0}=0.365 \sigma^{-3}$ $\left(\sigma_{\mathrm{He}}=2.556 \AA\right)$. The comparison of the equilibrium energy per particle of those two liquids, reported in Table 4 , revels that liquid ${ }^{4} \mathrm{He}$ is a more strongly self-bound liquid than liquid $\mathrm{T} \downarrow$. As it is expected, a smaller $e_{0}$ in case of liquid $T \downarrow$ is due to the smaller mass of $T \downarrow$ atoms and to the shallower $\mathrm{T} \downarrow-\mathrm{T} \downarrow$ interatomic potential.

Table 4. Comparison of the main relevant properties of liquids $\mathrm{T} \downarrow$ and ${ }^{4} \mathrm{He}$. The corresponding $\sigma\left(\sigma_{\mathrm{H}}=3.67 \AA\right.$ and $\left.\sigma_{\mathrm{He}}=2.556 \AA\right)$ are used for each liquid. The results for ${ }^{4}$ He are taken from Ref. 33

\begin{tabular}{l|c|c}
\hline \hline Parameter & $\mathrm{T} \downarrow$ & ${ }^{4} \mathrm{He}$ \\
\hline \hline$\rho_{0}, \sigma^{-3}$ & 0.369 & 0.365 \\
$e_{0}, \mathrm{~K}$ & -3.656 & -7.277 \\
$\rho_{S}, \sigma^{-3}$ & 0.277 & 0.264 \\
$P_{S}$, bar & -1.48 & -9.30 \\
\hline \hline
\end{tabular}

Knowing the equation of state $e(\rho)$ allows for the calculation of the pressure and the speed of sound (Eqs. (14) and (15)). Using those equations it is possible to determine the spinodal density $\rho_{s}$, i.e., the density at which the speed of sound becomes zero. The determination of the spinodal density is very important when quantum liquids are under investigation since at the spinodal pressure $P_{S}$ quantum liquids become macroscopically unstable with respect to density fluctuations. The instability of liquid $T \downarrow$ is defined by a small negative pressure, $P_{S}=-1.48(2)$ bar, at density $\rho_{s}=0.0056 \AA^{-3}=0.277 \sigma^{-3}$. If we compare those values with the values that define instability of liquid ${ }^{4} \mathrm{He}$, given in Ref. $33\left(\rho_{s}=0.264 \sigma^{-3}, P_{s}=-9.30(15)\right.$ bar $)$, we can say that spinodal densities of those two liquids are very similar in $\sigma$ units, while the spinodal pressure is slightly more negative in the case of liquid ${ }^{4} \mathrm{He}$. It is also worth mentioning that a previous approximate prediction of the liquid $\mathrm{T} \downarrow$ equilibrium density $\left(\rho_{0} \sim 0.0050 \AA^{-3}\right.$ ) given in Ref. 3, obtained with the VMC method and the Morse interaction potential, lies below the spinodal density obtained with the DMC method and the JDW interaction potential. The pressure and the speed of sound are plotted in Fig. 27 for liquid (dashed lines) and solid (solid lines) $\mathrm{T} \downarrow$. The results for the liquid phase include the density

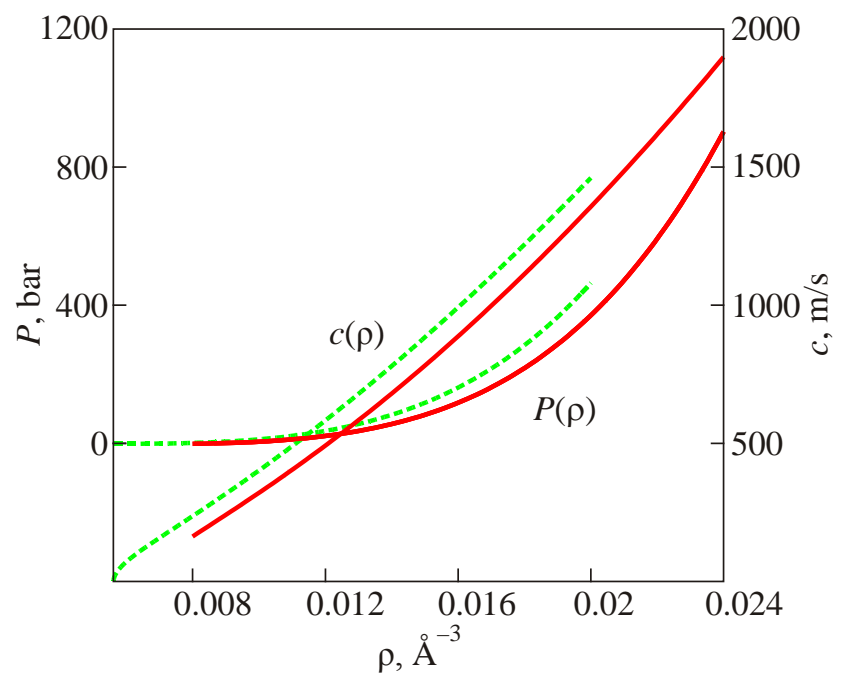

Fig. 27. (Color online) Pressure and speed of sound of liquid $\mathrm{T} \downarrow$ (dashed lines) and solid $\mathrm{T} \downarrow$ (solid lines) as a function of the density. Left (right) scale corresponds to pressure (speed of sound).

region from the spinodal density $\rho_{s}=0.0056 \AA^{-3}$ up to $\rho=0.02 \AA^{-3}$.

In addition to the energy profile of liquid $\mathrm{T} \downarrow$, the DMC method allows for determination of other ground-state quantities that describe the structural profile of the investigated liquid, such as the two-body radial distribution function $g(r)$ and the static structure factor $S(k)$. To avoid bias coming from the trial wave function used in the simulations the obtained $g(r)$ and $S(k)$ are calculated using pure estimators [32]. The two-body radial distribution function is given in Fig. 28 for several densities of liquid $\mathrm{T} \downarrow$. As it is expected, the strength of the main peaks increases with the density, and at the same time the main

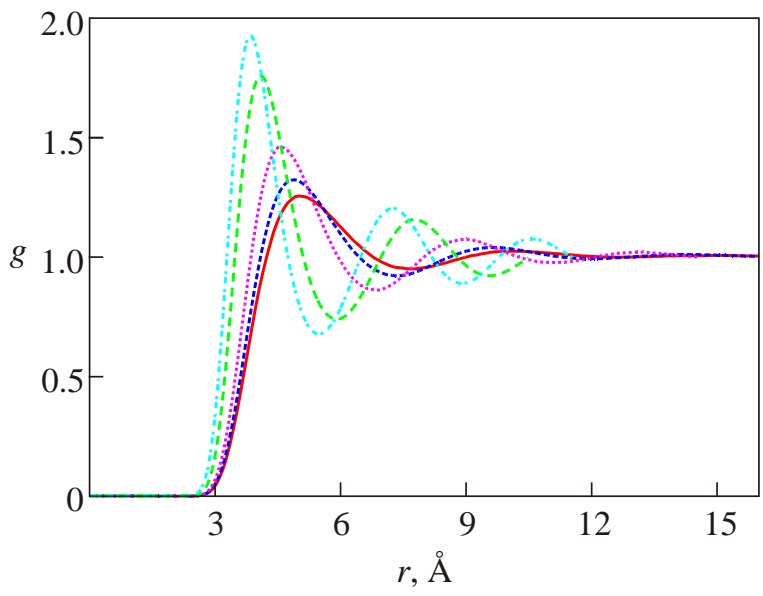

Fig. 28. (Color online) Two-body radial distribution functions of liquid $\mathrm{T} \downarrow$. From bottom to top in the height of the main peak, the results correspond to densities $0.006 \AA^{-3}$ (solid line), $0.0074 \AA^{-3}$ (short-dashed line), $0.01 \AA^{-3}$ (dotted line), $0.016 \AA^{-3}$ (longdashed line), and $0.02 \AA^{-3}$ (dashed-dotted line). 
peaks start to be localized at smaller interparticle distances. It is also evident that the increase of the density is accompanied with the appearance of secondary peaks at larger interparticle distances. The first appearance of a more pronounced second peak and the formation of a third peak can be seen at the density $\rho=0.01 \AA^{-3}$, which is very close to the freezing density. In the case of the highest density, $\rho=$ $=0.02 \AA^{-3}$, a pronounced structure is present. Since this density is above the freezing point, where the crystalline phase is more favorable, three visible peaks reflect the tendency towards spatial order in the system.

Three crystalline lattices, bcc, fcc and hcp, were used to investigate the solid phase of $\mathrm{T} \downarrow$. The obtained DMC results for the energy per particle at the different densities are shown in Fig. 29 for the selected densities in the range from 0.008 to $0.024 \AA^{-3}$. As can be seen from the figure, the energies per particle for all three lattices and for almost the whole region of the investigated densities, are practically the same within the statistical error. A slight difference between the results is present only when the system is very dense. Since the results obtained with the hcp lattice are slightly below the ones obtained with the bcc and fcc lattices, the hcp lattice was chosen as the one energetically preferred. Hereafter, we will refer to hcp lattice as the solid phase of $T \downarrow$. In accordance with that, the line shown on the top of the data in Fig. 29 is the equation of state of the solid hcp lattice, obtained by numerical fit to the DMC results using the analytical function

$$
e(\rho)=s_{2} \rho^{2}+s_{3} \rho^{3}+s_{4} \rho^{4}
$$

The best set of parameters that fit the DMC data is: $s_{2}=-10.47(11) \cdot 10^{4} \mathrm{~K} \cdot \AA^{2}, s_{3}=7.13(15) \cdot 10^{6} \mathrm{~K} \cdot \AA^{3}$, and $s_{4}=7.3(5) \cdot 10^{7} \mathrm{~K} \cdot \AA^{4}$. From the obtained equation of state the pressure and the speed of sound (Eqs. (14) and (15))

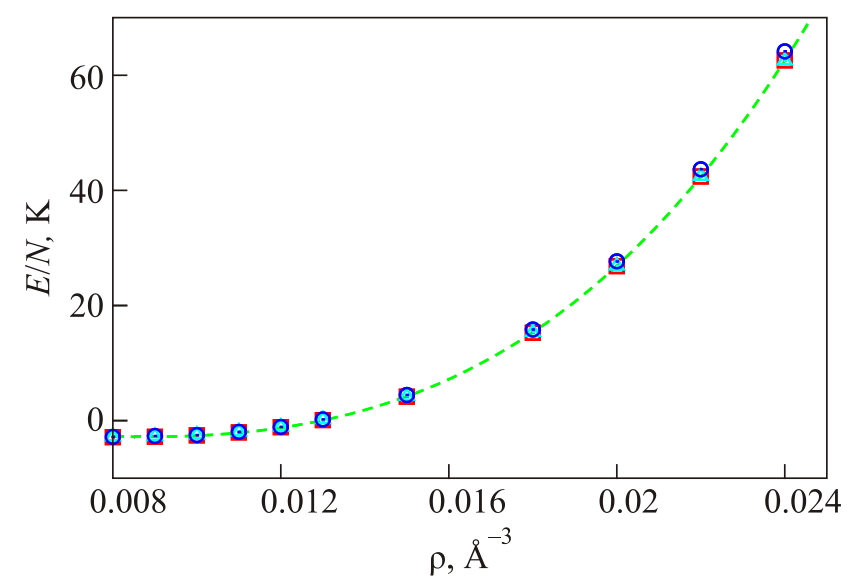

Fig. 29. (Color online) Energy per particle of solid $\mathrm{T} \downarrow$ as a function of the density $\rho$ for the hcp (boxes), fcc (triangles) and bcc (circles) lattices. The dashed line corresponds to the fit to the DMC energies using Eq. (22). The error bars of the DMC energies are smaller than the size of the symbols. were derived and plotted with solid lines in Fig. 27 for solid $T \downarrow$. The results include the density region used in the fitting of the solid phase equation of state, i.e., from the density $\rho=0.008 \AA^{-3}$ up to $\rho=0.024 \AA^{-3}$. The difference between the liquid and the solid phase properties of the system is also demonstrated through the different density dependence of $c(\rho)$ and $P(\rho)$ in both phases.

Results for $g(r)$ of the solid phase are reported in Fig. 30 for several selected densities. For all the shown densities pronounced structural profiles reflect the spatial ordering of the crystalline structure. The formation of the second and third peaks is clearly present in all cases, and the main peaks are higher than in the case of $g(r)$ of the liquid phase (Fig. 28). For example, if one compares results for the two-body radial distribution functions of the liquid phase (Fig. 28) and the solid phase (Fig. 30) at the density $\rho=0.02 \AA^{-3}$, one can note that the main peak is significantly higher in the case of the solid phase $\left(g\left(r_{m}\right) \simeq 2.23\right)$ than in the case of liquid $\left(g\left(r_{m}\right) \simeq 1.92\right)$. The overall tendency of the main peak shifting to smaller interparticle distances when the density increases, as well as the growth of its height, is observed for $g(r)$ of solid phase as well.

From the DMC results on the liquid and solid equations of state of $\mathrm{T} \downarrow$ one can study the liquid-solid phase transition. In order to localize the transition, the double-tangent Maxwell construction is used; the common tangent to both the liquid and solid equations of state is shown in Fig. 31. The intersections of the common tangent with the equations of state define the freezing $\left(\rho_{f}\right)$ and melting $\left(\rho_{m}\right)$ densities, marked with arrows in Fig. 31. The extracted numerical values, corresponding to a common pressure of the phase transition of $P=9(1)$ bar, are $\rho_{f}=0.00964 \AA^{-3}=$ $=0.477 \sigma^{-3}$ and $\rho_{m}=0.01069 \AA^{-3}=0.528 \sigma^{-3}$. The isotopic difference in masses between $\mathrm{T} \downarrow$ and $\mathrm{H} \downarrow$ atoms

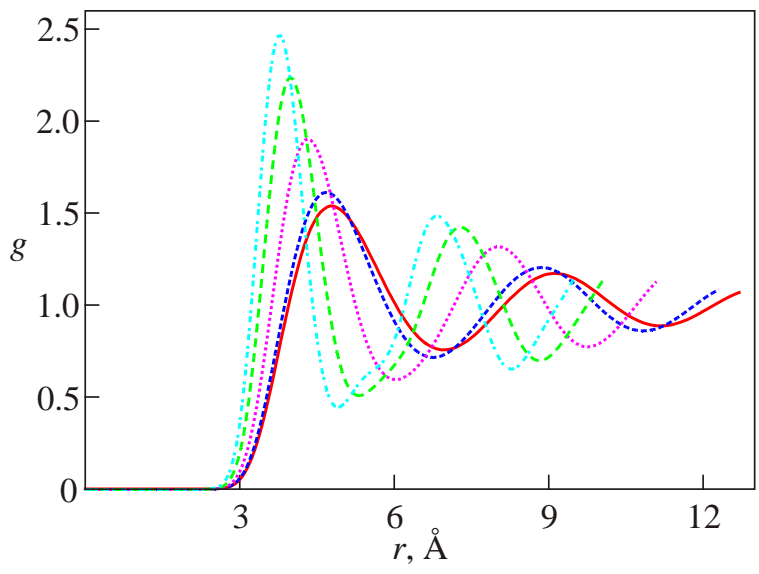

Fig. 30. (Color online) Two-body radial distribution functions of solid $\mathrm{T} \downarrow$. From bottom to top in the height of the main peak, the results correspond to densities $0.01 \AA^{-3}$ (solid line), $0.011 \AA^{-3}$ (short-dashed line), $0.015 \AA^{-3}$ (dotted line), $0.026 \AA^{-3}$ (longdashed line), and $0.024 \AA^{-3}$ (dashed-dotted line). 


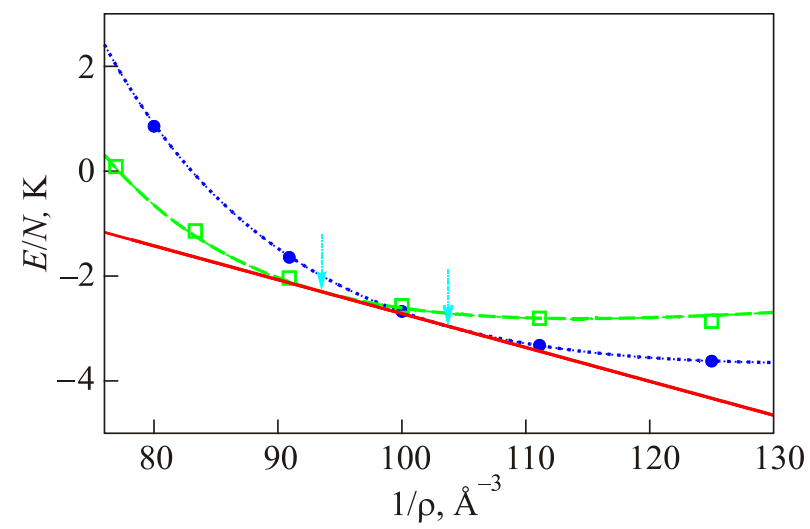

Fig. 31. (Color online) Maxwell construction based on plotting the energy per particle $E / N$ as a function of $1 / \rho$ in $T \downarrow$. The densities at which the first-order transition occurs are identified by finding the common tangent (solid line) to both the solid (dashed line) and liquid curve (dotted line).

causes the occurrence of the gas-solid phase transition in bulk $\mathrm{H} \downarrow$ at higher densities, $\rho_{f}=0.01328 \AA^{-3}=0.656 \sigma^{-3}$ and $\rho_{m}=0.01379 \AA^{-3}=0.682 \sigma^{-3}$, and considerable higher pressure $P=173(15)$ bar. Since in liquid ${ }^{4} \mathrm{He}$ the liquid-solid phase transition occurs at densities $\rho_{f}=0.430 \sigma^{-3}$ and $\rho_{m}=0.468 \sigma^{-3}$, and a common pressure of 25.3 bar [60], it can be noticed that the transition densities in liquid $\mathrm{T} \downarrow$ are closer to the liquid-solid transition densities in liquid ${ }^{4} \mathrm{He}$ (in $\sigma$ units) than to the gassolid transition densities in gas $\mathrm{H} \downarrow$.

Although the notable difference in $g(r)$ is observed at transition densities $\rho_{f}$ and $\rho_{m}$, we decided to plot in Fig. 32 the DMC results of the static structure factor $S(k)$ at transition densities, as a clear example of the difference between the two phases. Namely, the emergence of strong peaks at reciprocal-lattice sites characterizes $S(k)$ of the solid phase, while no peaks are observed in the case of the liquid phase.

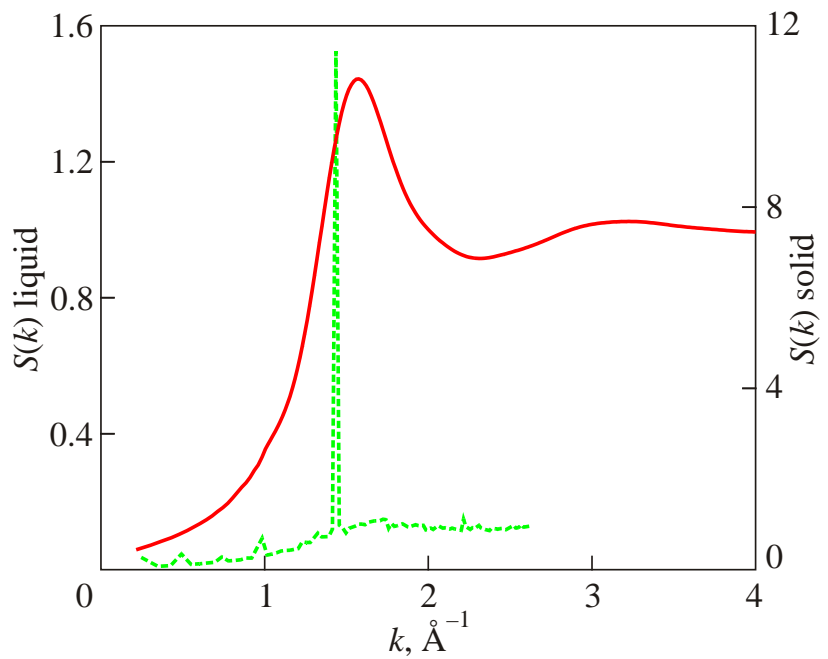

Fig. 32. (Color online) Static structure factor at the liquid-solid phase transition in $T \downarrow$. The results correspond to the liquid at $\rho_{f}$ (solid line) and to the solid at $\rho_{m}$ (dashed line).

\section{2. $\mathrm{T} \downarrow$ clusters}

Some ground-state liquid $\mathrm{T} \downarrow$ properties can been extrapolated from the results of the ground-state properties of $(\mathrm{T} \downarrow)_{N}$ clusters, where $N$ is the number of atoms in the cluster. The same procedure was successfully applied for ${ }^{4} \mathrm{He}$ and ${ }^{3} \mathrm{He}$ clusters, and the obtained equilibrium liquid ground-state properties were in good agreement with the experimental results [61,62]. An analytical expression of the liquid-drop formula

$$
E(N) / N=E_{v}+x E_{S}+x^{2} E_{C},
$$

reproduces well the energy per particle $E(N) / N$ of quantum liquid clusters if the variable $x$ is defined as $x=N^{-1 / 3}$. The coefficients $E_{v}, E_{s}$ and $E_{C}$ are the volume, surface and curvature terms, respectively.

To examine whether the liquid-drop formula can be applied to $(\mathrm{T} \downarrow)_{N}$ clusters, the DMC results for the energy per particle for clusters having from 20 to 320 atoms were fitted in to the liquid-drop model (23) (Ref. 28). The optimal fitting parameters are $E_{v}=-3.66(3) \mathrm{K}, E_{s}=$ $=10.2(2) \mathrm{K}$ and $E_{C}=-6.1(4) \mathrm{K}$, and they were obtained without including in the fit the equilibrium liquid $\mathrm{T} \downarrow$ energy per particle $e_{0}$. It was also checked that the parameters remain the same when the fitting is performed with $e_{0}$ included. In Fig. 33, the DMC results for the energy per particle in clusters $(\mathrm{T} \downarrow)_{N}, N=20$-320, as well as the solid line representing the numerical fit using the liquid-drop formula (23), are shown. The dashed line in Fig. 33 stands for the value $e_{0}$ obtained in bulk liquid $\mathrm{T} \downarrow$ (Ref. 24). Having in mind that the parameter $E_{v}$ represents the energy per particle of liquid $\mathrm{T} \downarrow$ at equilibrium density, it is evident that the obtained value $E_{v}=-3.66(3) \mathrm{K}$ is in a very good agreement with the result $e_{0}=-3.656(4) \mathrm{K}$ obtained in bulk liquid $\mathrm{T} \downarrow$ calculations.

Another quantity that can be compared with the one that came out from liquid $\mathrm{T} \downarrow$ study is the equilibrium density.

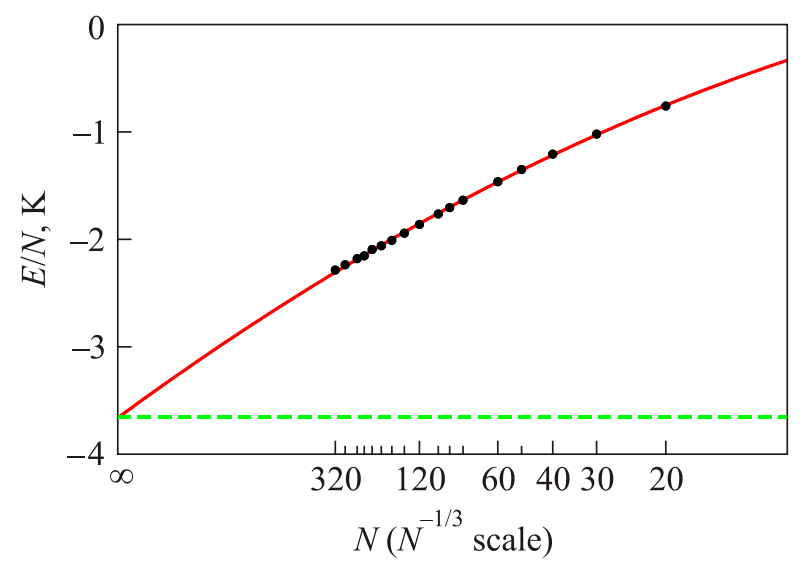

Fig. 33. (Color online) Energy per particle of $(\mathrm{T} \downarrow)_{N}$ clusters for $N=20-320$. Abscissa is $N$, on an $N^{-1 / 3}$ scale. The value of the energy per particle obtained in bulk liquid $T \downarrow$ study (Ref. 24) is plotted with a dashed line. 


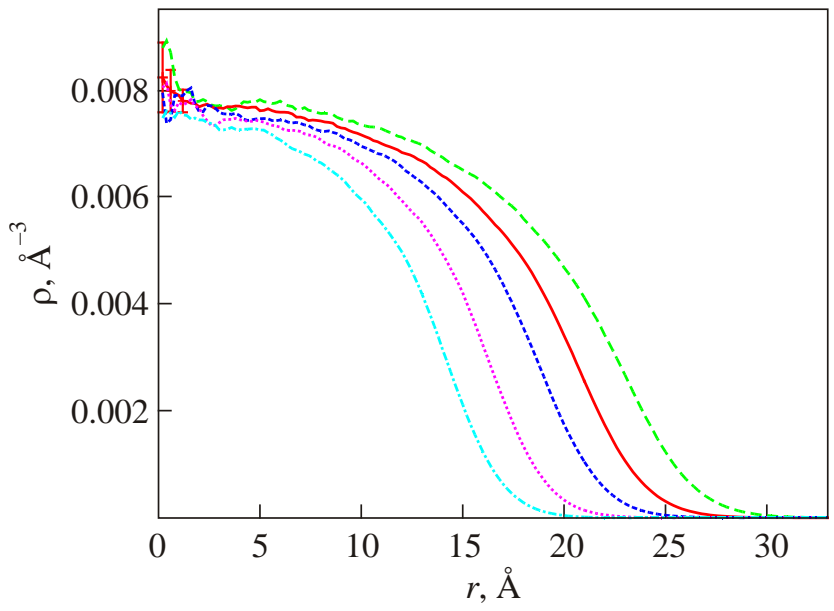

Fig. 34. (Color online) Density profiles for several $(\mathrm{T} \downarrow)_{N}$ clusters. Long-dashed, solid, short-dashed, dotted, and dashed-dotted lines correspond, respectively, to $N=320,240,180,120$, 80 . The error bars are large for small distances to the center of mass of the clusters, as indicated in the figure for the cluster having 240 atoms, and decrease for larger distances.

As we pointed out in the subsection concerning the liquid phase of bulk $T \downarrow$, the obtained equilibrium density is $\rho_{0}=0.007466(7) \AA^{-3}$. In the limit $N \rightarrow \infty$, the central density of cluster $(\mathrm{T} \downarrow)_{N}$ should approach the value $\rho_{0}$. In order to examine this assumption, the density profiles for several $\mathrm{T} \downarrow$ clusters are reported in Fig. 34. The plotted density profiles are obtained using pure estimators to exclude any bias coming from the trial wave function employed in simulations. Although the central densities have a huge noise at small distances, for the largest clusters they are very similar to the liquid equilibrium density $\rho_{0}$.

\section{Conclusions}

We have reviewed the theoretical research on spinpolarized atomic hydrogen and its isotopes in the last decade. The use of quantum Monte Carlo methods, nowadays one of the best possible approaches for correlated quantum many-body systems, and the knowledge of a very precise interatomic interaction potential enabled improvement in the theoretical description of these systems. The extreme quantum nature of spin-polarized hydrogen and its isotopes, in which Bose and Fermi statistics play an important role, leads to a rich class of quantum phases. Spin-polarized hydrogen, the only system which remains in the gas phase at zero temperature, displays universal behavior at very low densities. By increasing the density $\mathrm{H} \downarrow$ solidifies at a pressure of $\sim 170$ bar. Universal behavior is also observed in low density two-dimensional $\mathrm{H} \downarrow$. Upon increase of the density, two-dimensional $\mathrm{H} \downarrow$ freezes in a triangular solid. Adsorbed on the surface of liquid ${ }^{4} \mathrm{He}, \mathrm{H} \downarrow$ forms a stable nearly two-dimensional quantum gas. However, a purely two-dimensional model for the layer is appropri- ate only for very low coverages. In particular, the condensate fraction of the layer is appreciably higher than in 2D.

The study of spin-polarized deuterium revealed different possibilities of its ground-state phases, depending on how $\mathrm{D} \downarrow$ atoms are distributed with respect to the available nuclear spin states. At zero pressure, $\mathrm{D} \downarrow_{3}$ and $\mathrm{D} \downarrow_{2}$ are quantum liquids, while $\mathrm{D} \downarrow_{1}$ shows the peculiar property of a gas-liquid zero-temperature phase transition. Spin-polarized tritium was studied in its ground state and the conditions under which it undergoes a liquid-solid phase transition were determined. It was proven that its liquid phase is less self-bound than liquid ${ }^{4} \mathrm{He}$ because of its shallower interaction.

The description of $\mathrm{H} \downarrow$ and its isotopes at finite temperature is still not complete, as only approximate calculations of $\mathrm{H} \downarrow$ at very low densities were done so far. Excitations have not been studied either and interesting questions regarding the universality of excitations at low temperatures, which are also relevant for the community of cold Bose gases, remain to be analyzed.

From the experimental point of view, trapping of spinpolarized hydrogen and reaching quantum degeneracy limits has been a great challenge. The new technique of trapping hydrogen and its isotopes, using Zeeman deceleration $[11,12]$, might in future enable observation of the theoretically predicted phases as well as the larger interplay between theory and experiment.

\section{Acknowledgments}

We would like to acknowledge the work of our colleagues J. Casulleras, J.M. Marin, and P. Stipanović, who also contributed to investigations presented within this review. L.V.M. and I.B. would like to express their gratitude to Professor S. Kilić who always strongly encouraged their work. We acknowledge partial financial support from the DGI (Spain) Grant No. FIS2011-25275, the Generalitat de Catalunya Grant No. 2009SGR-1003, and the MSES (Croatia) Grant No. 177-1770508-0493. These materials are based on work financed by the Croatian Science Foundation and I.B. acknowledges the support. In addition, the resources of the Zagreb University Computing Centre (Srce) and Croatian National grid Infrastructure (CRO NGI) were used, as well as the resources of the HYBRID cluster at the University of Split, Faculty of Science.

1. W. Kolos and L. Wolniewicz, J. Chem. Phys. 43, 2429 (1965); Chem. Phys. Lett. 24, 457 (1974).

2. J.V. Dugan, Jr., and R.D. Etters, J. Chem. Phys. 59, 6171 (1973).

3. R.D. Etters, J.V. Dugan, Jr., and R.W. Palmer, J. Chem. Phys. 62, 313 (1975).

4. W.C. Stwaley and L.H. Nosanow, Phys. Rev. Lett. 36, 910 (1976).

5. I.F. Silvera and J.T.M. Walraven, Phys. Rev. Lett. 44, 164 (1980). 
6. D.G. Fried, T.C. Killian, L. Willmann, D. Landhuis, S.C. Moss, D. Kleppner, and T.J. Greytak, Phys. Rev. Lett. 81, 3811 (1998).

7. T.J. Greytak, D. Kleppner, D.G. Fried, T.C. Killian, L. Willmann, D. Landhuis, and S.C. Moss, Physica B 280, 20 (2000).

8. A.I. Safonov, S.A. Vasilyev, I.S. Yasnikov, I.I. Lukashevich, and S. Jaakkola, Phys. Rev. Lett. 81, 4545 (1998).

9. J. Järvinen and S. Vasilyev, J. Phys.: Conf. Series 19, 186 (2005).

10. J. Järvinen, J. Ahokas, S. Jaakkola, and S.A. Vasilyev, Phys. Rev. A 72, 052713 (2005).

11. A.W. Wiederkehr, S.D. Hogan, B. Lambillotte, M. Andrist, H. Schmutz, J. Agner, Y. Salathé, and F. Merkt, Phys. Rev. A 81, 021402(R) (2010).

12. S.D. Hogan, A.W. Wiederkehr, H. Schmutz, and F. Merkt, Phys. Rev. Lett. 101, 143001 (2008).

13. M.D. Miller and L.H. Nosanow, Phys. Rev. B 15, 4376 (1976).

14. L.J. Lantto and R.M. Nieminen, J. Low Temp. Phys. 37, 1 (1979).

15. P. Entel and J. Anlauf, Z. Phys. B: Condens. Matter 42, 191 (1981).

16. E. Krotscheck, R.A. Smith, J.W. Clark, and R.M. Panoff, Phys. Rev. B 24, 6383 (1981).

17. R.M. Panoff, J.W. Clark, M.A. Lee, K.E. Schmidt, M.H. Halos, and G.V. Chester, Phys. Rev. Lett. 48, 1675 (1982).

18. M.F. Flynn, J.W. Clark, E. Krotscheck, R.A. Smith, and R.M. Panoff, Phys. Rev. B 32, 2945 (1985).

19. R.M. Panoff and J.W. Clark, Phys. Rev. B 36, 5527 (1987).

20. B. Skjetne and E. Østgaard, J. Phys.: Condens. Matter 11, 8017 (1999).

21. J.H. Freed, J. Chem. Phys. 72, 1414 (1980).

22. M.J. Jamieson, A. Dalgarno, and L. Wolniewicz, Phys. Rev. A 61, 042705 (2000).

23. L. Vranješ Markić, J. Boronat, and J. Casulleras, Phys. Rev. B 75, 064506 (2007).

24. I. Bešlić, L. Vranješ Markić, and J. Boronat, Phys. Rev. B 80, 134506 (2009).

25. I. Bešlić, L. Vranješ Markić, J. Cassuleras, and J. Boronat, Phys. Rev. B 88, 024507 (2013).

26. L. Vranješ Markić and J. Boronat, J. Low Temp. Phys., in press (2012).

27. J.M. Marín, L. Vranješ Markić, and J. Boronat, submitted to J. Chem. Phys.

28. I. Bešlić, L. Vranješ Markić, and J. Boronat, J. Chem. Phys. 131, 244506 (2009).

29. I. Bešlić, L. Vranješ Markić, J. Casulleras, and J. Boronat, J. Low Temp. Phys. 171, 436 (2013).

30. J. Mullin, Phys. Rev. 134, A1249 (1964); L.H. Nosanow, Phys. Rev. Lett. 13, 270 (1964).

31. J. Boronat and J. Casulleras, Phys. Rev. B 49, 8920 (1994).

32. J. Casulleras and J. Boronat, Phys. Rev. B 52, 3654 (1995).

33. J. Boronat, Monte Carlo Simulations at Zero Temperature: Helium in One, Two and Three Dimensions, Series on Advances in Quantum Many-Body Theory, Vol. 4, Microscopic approaches to quantum liquids in confined geometries, Chapter 2, World Scientific (2004).
34. L. Vranješ, J. Boronat, J. Casulleras, and C. Cazorla, Phys. Rev. Lett. 95, 145302 (2005).

35. W. Kolos and J. Rychlewski, J. Mol. Spectrosc. 143, 237 (1990).

36. I.F. Silvera and V.V. Goldman, Phys. Rev. Lett. 45, 915 (1980).

37. D. Blume, B.D. Esry, C.H. Greene, N.N. Klausen, and G.J. Hanna, Phys. Rev. Lett. 89, 163402 (2002).

38. B.R. Joudeh, M.K. Sugheir, and H.B. Ghassib, Physica B 388, 237 (2007).

39. I. Bešlić, L. Vranješ Markić, and J. Boronat, J. Chem. Phys. 128, 064302 (2008).

40. I. Bešlić, L. Vranješ Markić, and J. Boronat, J. Phys.: Conf. Series 150, 032010 (2009).

41. P. Stipanović, L. Vranješ Markić, J. Boronat, and B. Kehić, J. Chem. Phys. 134, 054509 (2011).

42. Zong-Chao Yan, James F. Babb, A. Dalgarno, and G.W.F. Drake, Phys. Rev. A 54, 2824 (1996).

43. R.A. Aziz, F.R.W. McCourt, and C.C.K. Wong, Mol. Phys. 61, 1487 (1987).

44. G. Das, A.F. Wagner, and A.C. Wahl, J. Chem. Phys. 68, 4917 (1978).

45. N.N. Bogoliubov, J. Phys. (U.S.S.R.) 11, 23 (1947).

46. T.D. Lee, K. Huang, and C.N. Yang, Phys. Rev. 106, 1135 (1957).

47. S. Giorgini, J. Boronat, and J. Casulleras, Phys. Rev. A 60, 5129 (1999); J. Boronat, J. Casulleras, and S. Giorgini, Physica B 284, 1 (2000).

48. Carl J. Williams and Paul S. Julienne, Phys. Rev. A 47, 1524 (1993).

49. C. Pierleoni, D.M. Ceperley, and M. Holzmann, Phys. Rev. Lett. 93, 146402 (2004).

50. R.L. Danilowicz, J.V. Dugan, Jr., and R.D. Etters, J. Chem. Phys. 65, 498 (1976).

51. M.K. Al-Sugheir, A.S. Sandouqa, B.R. Joudeh, S. Al-Omari, M. Awawdeh, and F. Rawwagah, Physica B 405, 2171 (2010).

52. S. Pilati, J. Boronat, J. Casulleras, and S. Giorgini, Phys. Rev. A 71, 023605 (2005).

53. M. Schick, Phys. Rev. A 3, 1067 (1971).

54. M.K. Al-Sugheir, H.B. Ghassib, and M. Awawdeh, Phys. Rev. A 84, 013617 (2011).

55. I.B. Mantz and D.O. Edwards, Phys. Rev. B 20, 4518 (1979).

56. J.M. Marín, J. Boronat, and J. Casulleras, Phys. Rev. B 71, 144518 (2005).

57. R. Guardiola and J. Navarro, Phys. Rev. Lett. 89, 193401 (2002).

58. J.W. Clark and R.M. Panoff, Condensed Matter Theories, Vol. 4, J. Keller (ed.), Plenum, New York (1989), p. 1.

59. V.N. Efimov and M.Ya. Amusya, Sov. Phys. JETP 20, 388 (1965).

60. D.O. Edwards and R.C. Pandorff, Phys. Rev. A 140, 816 (1965).

61. V.R. Pandharipande, S.C. Pieper, and R.B. Wiringa, Phys. Rev. B 34, 4571 (1986).

62. S.A. Chin and E. Krotscheck, Phys. Rev. B 45, 852 (1992). 